\title{
Brainstem Neurons that Command Left/Right Locomotor Asymmetries
}

\author{
Jared M. Cregg ${ }^{1}$, Roberto Leiras ${ }^{1}$, Alexia Montalant ${ }^{1}$, lan R. Wickersham ${ }^{2}$, and Ole Kiehn ${ }^{1,3, *}$ \\ ${ }^{1}$ Department of Neuroscience, University of Copenhagen, 2200 Copenhagen, Denmark \\ ${ }^{2}$ The McGovern Institute for Brain Research, Massachusetts Institute of Technology, Cambridge, Massachusetts 02139, USA \\ ${ }^{3}$ Department of Neuroscience, Karolinska Institutet, 17177 Stockholm, Sweden \\ *Corresponding author
}

\begin{abstract}
Descending command neurons instruct spinal networks to execute basic locomotor functions, such as which gait and what speed. The command functions for gait and speed are symmetric, implying that a separate unknown system directs asymmetric movements - the ability to move left or right. Here we report the discovery that $\mathrm{Chx}$ 10-lineage reticulospinal neurons act to control the direction of locomotor movements in mammals. Chx10 neurons exhibit ipsilateral projection, and can decrease spinal limb-based locomotor activity ipsilaterally. This circuit mechanism acts as the basis for left or right locomotor movements in freely moving animals: selective unilateral activation of Chx10 neurons causes ipsilateral movements whereas inhibition causes contralateral movements. Spontaneous forward locomotion is thus transformed into an ipsilateral movement by braking locomotion on the ipsilateral side. We identify sensorimotor brain regions that project onto $C h x 10$ reticulospinal neurons, and demonstrate that their unilateral activation can impart left/right directional commands. Together these data identify the descending motor system which commands left/right locomotor asymmetries in mammals.
\end{abstract}

Keywords: spinal cord, locomotion, motor control, brainstem, superior colliculus

\section{INTRODUCTION}

Locomotion is a natural behavior universal to the animal kingdom. In vertebrates, coordination of rhythmic locomotor movements occurs largely within circuits of the spinal cord itself (1-7). For these circuits to function, they need commands from supraspinal effector neurons that control the start and speed of locomotion. The brainstem command neurons which control these parameters have been examined extensively in several vertebrate species (8-20). Recently, brainstem neurons that mediate locomotor stop were also identified $(9,11,15)$.

Characteristically, when start command pathways of the midbrain locomotor region are activated unilaterally, they cause bilateral full-bodied locomotion which proceeds in a straight line $(10,11,14,17,18,20-23)$. This finding underscores symmetry in the command for initiating locomotion and controlling its speed. The anatomical basis for this symmetry has been worked out in some detail. At the level of the midbrain locomotor region, which is comprised of glutamatergic neurons in the cuneiform and pedunculopontine nuclei, neurons exhibit extensive commissural connectivity with neurons of the contralateral side $(10,19)$. The start command is then relayed to reticulospinal neurons, including those of the lateral paragigantocellular nucleus (LPGi), which in turn activate spinal locomotor circuits $(11,21,24-$ $29)$. These reticulospinal neurons receive bilateral projections from the midbrain locomotor region $(10,11,21,23)$. Neurons of the LPGi project bilaterally, innervating both sides of the spinal cord, and unilateral optogenetic activation of LPGi glutamatergic neurons initiates symmetric fullbodied locomotion (11). Unilateral stimulation of neurons in the parapyramidal area also causes symmetric locomotor activity in (16).

A considerable gap in our knowledge is the inability to explain how command neurons direct locomotor movements to the left or right side (30). A system which executes left/right locomotor asymmetries would be required for any goal-directed locomotor movement, as might occur during basic behaviors like foraging, navigation, and/or escape, but also during specialized locomotor tasks (31). From in vitro studies, it is clear that rhythmogenic modules can operate independently within the left or right spinal cord in mammals (32-38). Nonetheless, differential engagement of left/right rhythmogenic modules cannot be mediated by a symmetric start command. Moreover, unilateral lesion of the corticospinal tract (i.e. unilateral pyramidotomy) does not result in any overt left/right locomotor asymmetries $(39,40)$. Although unilateral labyrinth ablation or vestibulocochlear nerve (VIII) stimulation can initiate reflexive rotational behavior (41), this self-righting reflex is not a voluntary locomotor command.

In the present work, we hypothesized that a turn in limbed animals could be implemented by inhibition of locomotor circuits on one side. We focused on a system of nucleus gigantocellularis $(\mathrm{Gi})$ reticulospinal neurons uniquely identified by expression of ChxlO (9). These Chxl0 'stop neurons' are glutamatergic, and their bilateral activation arrests locomotion by suppressing locomotor rhythmogenesis in the spinal cord (9). We found that Chx10 Gi neurons 1) exhibit dominant unilateral projection to the spinal cord that can bias locomotor activity unilaterally, 2) define the direction of locomotion by effecting changes in ipsilateral limb and axial movements in vivo, and 3) can be engaged to impart asymmetric movements via unilateral input from distinct sensorimotor brain 
A

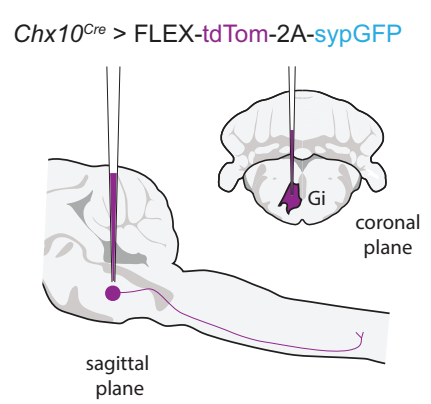

E
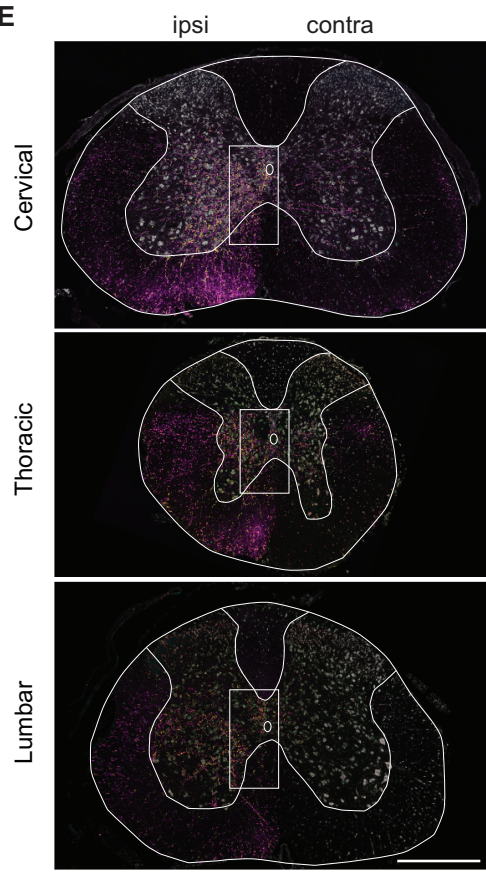

Nissl tdTom synGFP
B

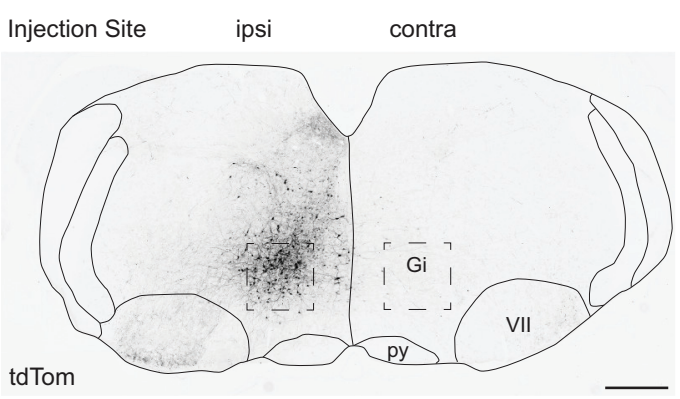

$\mathbf{F}$
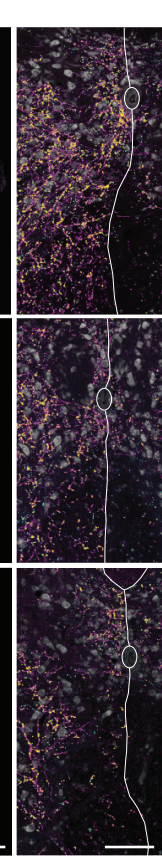

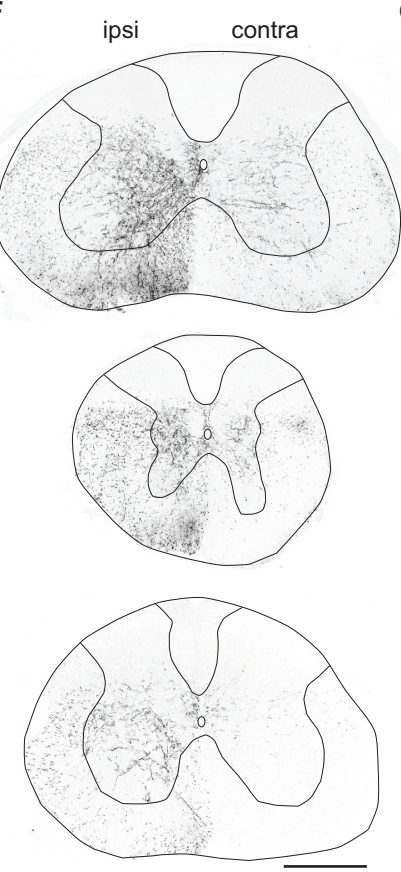

tdTom

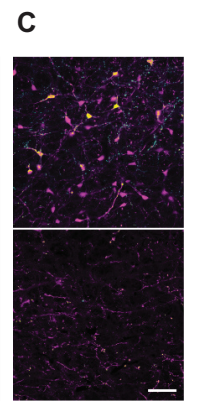

tdTom sypGFP

G

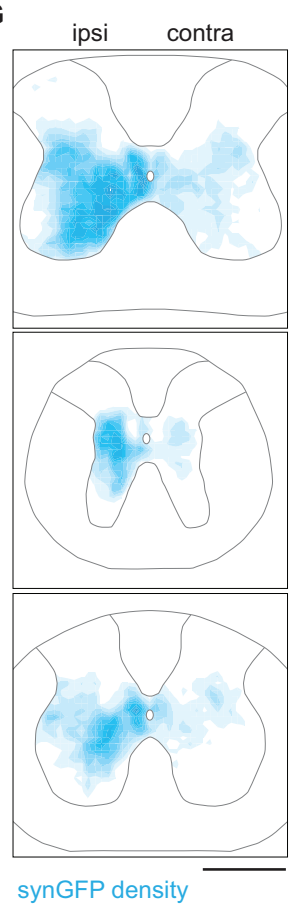

D

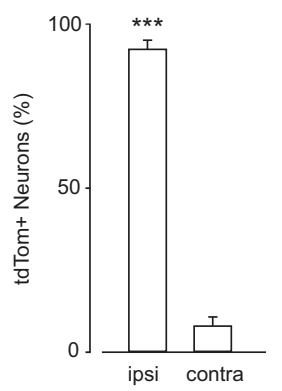

H
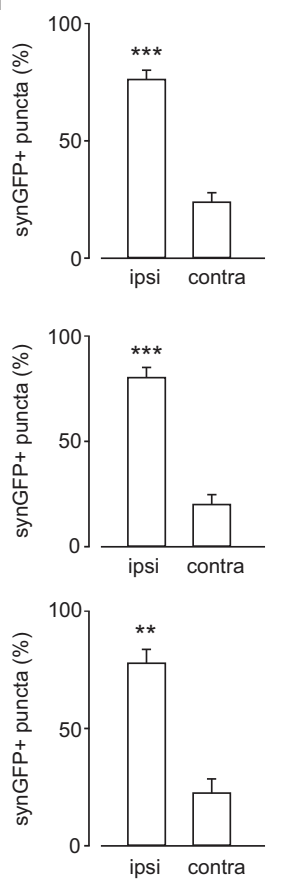

Figure 1. Chx10 Gi neurons form a prominent tract of descending axons that project ipsilaterally. A, Unilateral labeling of $C h \times 10$ neurons of the rostral gigantocellularis (Gi) using the Cre-dependent anterograde tracer AAV-FLEX-tdTom-2A-synGFP. B, Inverted fluorescent image of tdTomato ${ }^{+}$neurons at the injection site within Gi. C, Top, tdTom ${ }^{+} /$synGFP $^{+}$neurons at injection site. Bottom, sparse tdTom ${ }^{+}$axonal labeling and synGFP ${ }^{+}$punctae contralateral to the injection site. Scale bar $=50 \mu \mathrm{m}$. D, Quantification of tdTom ${ }^{+}$ neurons labeled ipsilateral (ipsi) and contralateral (contra) to the injection site. ${ }^{* *} P=3 \times 10^{-5}$, two-tailed t-test, $n=3$. E, Left, A prominent tract of tdTom ${ }^{+}$axons is observed within the ventrolateral funiculus of the cervical (top), thoracic (middle), and lumbar (bottom) spinal cord. Axons project almost exclusively on the ipsilateral side. SynGFP ${ }^{+}$punctae (overlap of cyan with magenta yields yellow) exhibit a high density on the ipsilateral side. Scale bar $=500 \mu \mathrm{m}$. Right, Insets from images on left demonstrate a sharp division in the density of axon profiles and synGFP punctae between the ipsilateral and contralateral sides. Scale bar $=100 \mu \mathrm{m}$. F, Inverted fluorescent images of tdTom ${ }^{+}$axonal projections in the cervical (top), thoracic (middle), and lumbar (bottom) spinal cord. Axons descend in the ventrolateral funiculus within a well-defined "wedge". Scale bar $=500 \mu \mathrm{m}$. G, Density plots for synGFP ${ }^{+}$punctae within the gray matter of the cervical (top), thoracic (middle), and lumbar (bottom) spinal cord (average from 3 animals). The density of putative synapses is greatest in the ipsilateral intermediate gray matter-laminae VII, VIII, and X. SynGFP ${ }^{+}$punctae were excluded from laminae I-VI of the dorsal horn, lamina IX (motor neurons), and at the thoracic level, Clarke's column. Scale bar $=500 \mu \mathrm{m}$. H, Quantification of synGFP punctae within the ipsilateral and contralateral spinal cord gray matter. Cervical, ${ }^{\star \star \star} P=0.0006$; Thoracic, ${ }^{\star \star *} P=0.0002$; Lumbar, ${ }^{\star \star} P=$ 0.007. Two-tailed t-test, $n=3$.

regions. Chx10 Gi neurons exhibit all the features of a bona fide system for executing left/right locomotor behaviors in limbed animals.

\section{RESULTS}

\section{Chx10 Gi neurons form a prominent spinal tract of ip- silaterally projecting axons.}

For Chx10 Gi neurons to regulate locomotion unilaterally, they should exhibit predominant unilateral projection to the spinal cord. We examined this by labeling Chxl0-lineage neurons of the rostral gigantocellularis with an anterograde 
A

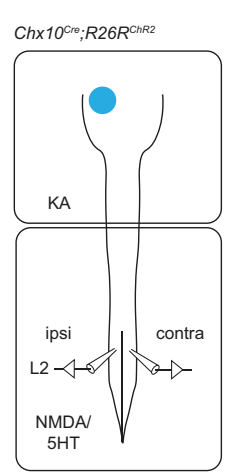

B

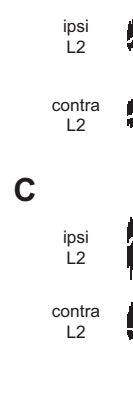

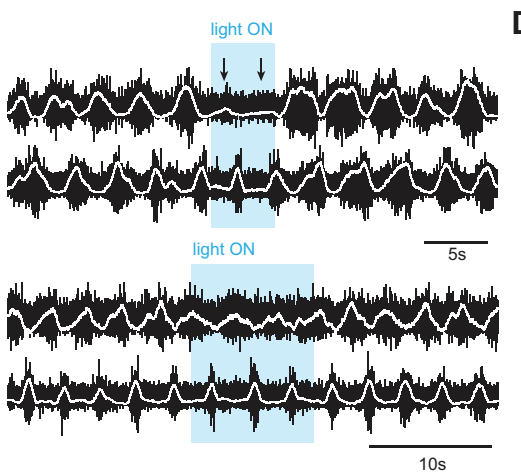

D

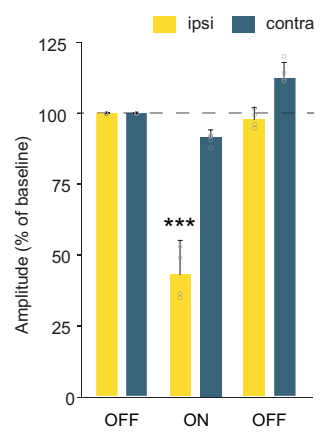

Figure 2. Chx10 Gi neurons can arrest and/or decrease in vitro locomotor-like activity on the same side. A, Schematic of split-bath brainstem-spinal cord preparation from $\mathrm{P} 0-2 \mathrm{Ch} \times 10^{\mathrm{Cre}} ; R 26 R^{\mathrm{ChR} 2}$ mice, which was used to interrogate unilateral function of Chx10 reticulospinal neurons in vitro. A rostral brainstem compartment was bathed with kynurenic acid (4 mM) to block all glutamatergic transmission amongst axon collaterals (42), and a caudal spinal cord compartment was bathed with $5 \mathrm{HT}(8 \mu \mathrm{M})$ and NMDA (8 $\mu \mathrm{M})$ to induce locomotor-like activity, assayed by recording from the $\mathrm{L} 2$ ventral roots. B, Unilateral photostimulation of $C h \times 10$ Gi neurons can arrest locomotor-like activity ipsilateral (but not contralateral) to stimulation. C, In another example, unilateral photostimulation of $C h x 10$ Gi neurons decreases the amplitude of motor bursts ipsilateral (but not contralateral) to stimulation. D, Quantification of burst amplitude before, during, and after photostimulation. ${ }^{* *} P<1.7 \times 10^{-4}$, one-way ANOVA, $n=4$.

viral tracer (Chx10 $0^{\mathrm{Cre}}>\mathrm{AAV}$-FLEX-tdTomato-2A-synGFP) (Figure 1A). In adult mice, a unilateral injection labeled Chxl0 Gi neurons predominantly on the same side (Figures 1B-1D). Chx10 neurons projected axons caudally (Figures S1A and S1B), which then coalesced to form a prominent ipsilateral tract of axons lateral to the inferior olive (Figure S1C). At the level of the pyramidal decussation, this tract of Chx10 reticulospinal axons turned ventrally to occupy the ipsilateral ventral funiculus within the rostral-most segment of the spinal cord (Figure S1C). TdTomato ${ }^{+}$axons arborized predominately within the ipsilateral cord (Figures $1 \mathrm{E}$ and $1 F)$, although some arborizations could also be found on the contralateral side.

We quantified the position and density of Chxlo Gi synGFP+ punctae (putative synapses) within the spinal cord. A vast majority of synGFP ${ }^{+}$punctae were ipsilateral to the injection site in the cervical, thoracic, and lumbar cord (76.3$80.6 \%$, Figures $1 \mathrm{G}$ and $1 \mathrm{H}$ ), with fewer synGFP ${ }^{+}$punctae on the contralateral side. The density of $\operatorname{synGFP}^{+}$punctae was greatest in the ipsilateral intermediate gray (laminae VII, VIII, and $\mathrm{X}$ ) (Figures $1 \mathrm{G}$ and $1 \mathrm{H}$ ), with the exception of Clarke's column (Th1 to L3) (Figures $1 \mathrm{G}$ and $1 \mathrm{H}$ ), a medial nucleus which conveys proprioceptive inputs to the cerebellum. Notably, synGFP ${ }^{+}$punctae were largely absent from dorsal horn laminae I-VI where sensory networks are localized, and from lamina IX where motor neurons reside. These data indicate that $C h x 10$ reticulospinal neurons likely act on rhythmic premotor networks $(7,9)$, rather than on motor neurons themselves (9). We conclude that $C h x 10 \mathrm{Gi}$ neurons have predominantly ipsilateral projections to the spinal cord, a feature that may allow for differential control of left and right spinal motor networks.

Chx10 Gi neurons enable differential control of rhythmic motor activity on the left and right sides.
We next asked whether ipsilateral projection of $C h \times 10 \mathrm{Gi}$ neurons can allow for differential control of left and right spinal motor networks using an in vitro split-bath preparation (Figure 2A). Here, locomotor-like activity is maintained in the spinal cord and synaptic activity is blocked in the brainstem. Stimulating Chx10 Gi neurons in this configuration excludes the possibility that locomotor effects are due to axon collaterals in the brainstem $(9,42)$. In $C h x 10^{C r e} ; R 26 R^{C h R 2}$ preparations, we found that unilateral stimulation of Chx10 Gi neurons with blue light could arrest rhythmic hindlimb locomotor activity only on the side ipsilateral to stimulation with little effect on the rhythm of the contralateral side (Figure $2 \mathrm{~B}, n=2 / 4)$. In other examples, we found a decrease in the amplitude of locomotor bursting ipsilateral to photostimulation (Figure 2C). Altogether, burst amplitude exhibited a $57 \pm 12 \%$ reduction ipsilateral (but not contralateral) to photostimulation (Figure 2D). These observations phenocopy data demonstrating that rhythmicity can be initiated on one side of the cord $(34,43)$, and that unilateral stimulation of inhibitory neurons in the lumbar spinal cord can slow or arrest locomotor rhythmogenesis only on the side of stimulation (34). We conclude that ChxlO reticulospinal neurons can function as a unilateral locomotor effector.

\section{Unilateral excitation of Chx10 Gi neurons in vivo re- veals a role in control of left/right movements.}

The capacity of Chx10 Gi neurons to act as an ipsilateral locomotor effector in vitro suggests a function of ChxlO Gi neurons in controlling locomotor asymmetries-the ability to move left or right. To test this, we examined the behavioral consequence of unilateral stimulation of Chxlo Gi neurons in freely moving mice. We expressed excitatory hM3Dq-DREADDs in Chxl0 Gi neurons on one side of the brainstem (Figure 3A). hM3Dq-DREADDs can be activated with low doses $\left(0.5 \mathrm{mg} \mathrm{kg}^{-1}\right)$ of clozapine-N-oxide (CNO), which causes neuronal depolarization via $\mathrm{G}_{\mathrm{q}}$-mediated signaling (44). In a cylinder assay, which promotes locomotor 
A Chx10 cre $>$ FLEX-hM3Dq-mCherry

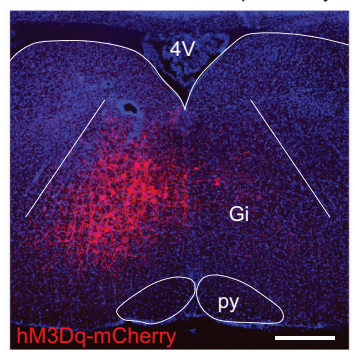

E

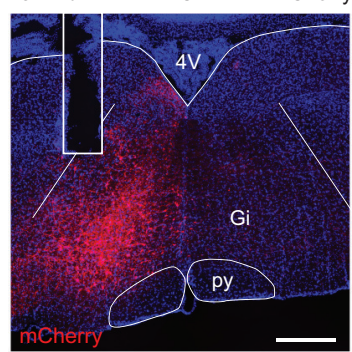

H

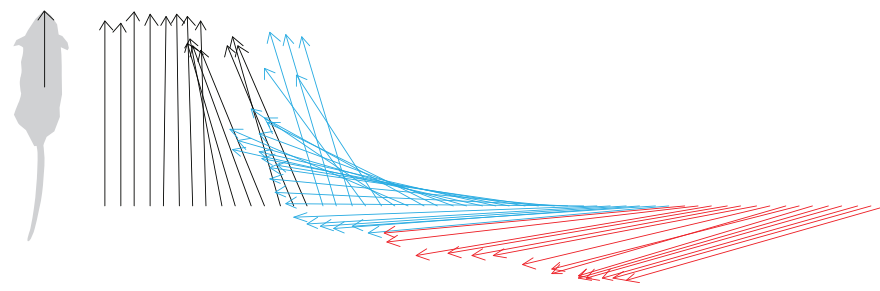

$0.6 \mathrm{~s}$ pre $1 \mathrm{~s}$ light $0.6 \mathrm{~s}$ post
B

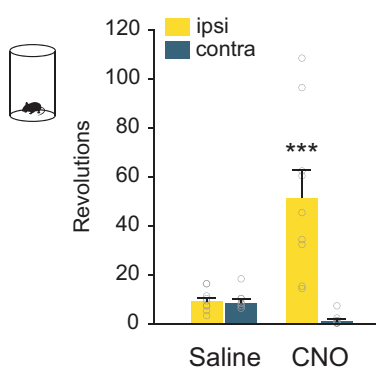

F
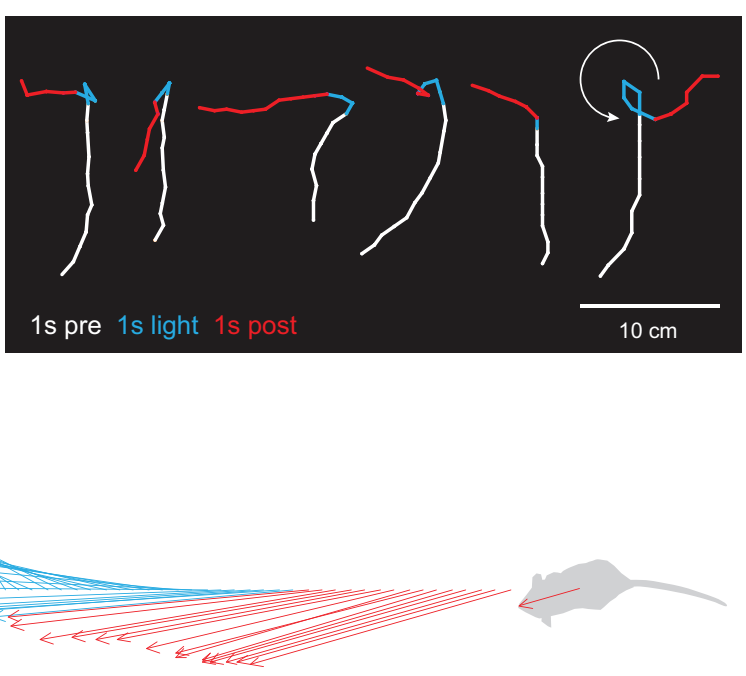

$\overline{200 \mathrm{~ms}}$
C
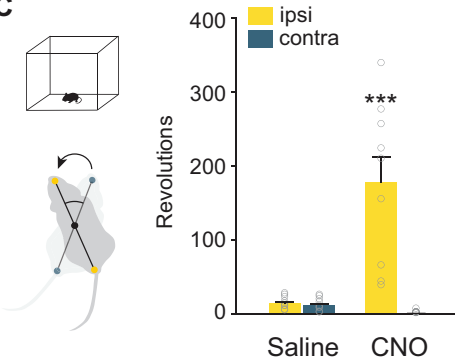

D

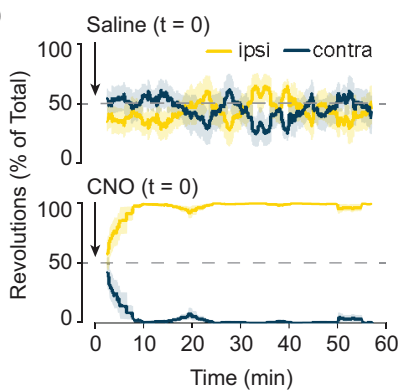

G
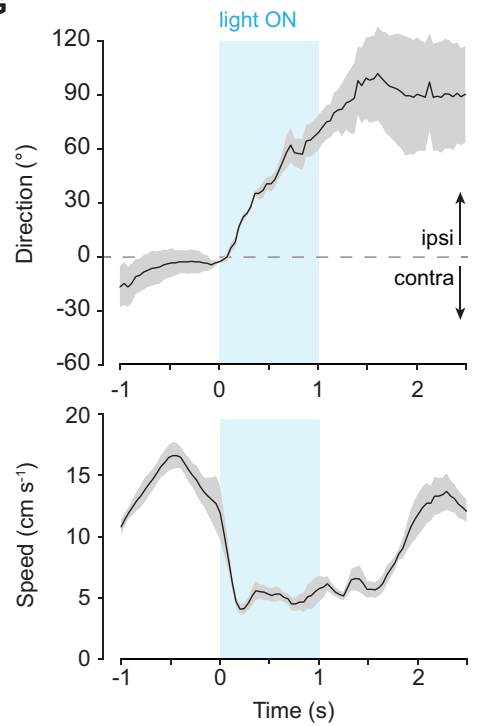

Figure 3. Excitation of $C h \times 10$ Gi neurons causes ipsilateral movements. A, Unilateral infection of Chx10 Gi neurons with AAVFLEX-hM3Dq-mCherry. Scale bar $=500 \mu \mathrm{m}$. B, Movement preference in a 10 minute cylinder assay $1 \mathrm{~h}$ after injection of saline or CNO. ${ }^{* * *} P<4 \times 10^{-5}$, one-way ANOVA with Tukey HSD, $n=9$. C, Movement preference in an open field assay, quantified between 30-60 min post-injection of saline or CNO. ${ }^{* * *} P<3 \times 10^{-6}$, one-way ANOVA with Tukey HSD, $n=9$. D, Instantaneous analysis of movement preference, quantified as the percentage of ipsilateral versus contralateral revolutions (bin = $5 \mathrm{~min}$ ), following injection of saline (top) or CNO (bottom) at $\mathrm{t}=0$. E, Unilateral injection of AAV-FLEX-ChR2-2A-mCherry in Chx10 Cre mice. Example of mCherry expression and optical fiber placement in Gi. Scale bar $=500 \mu \mathrm{m}$. F, Body tracking $1 \mathrm{~s}$ before (white), $1 \mathrm{~s}$ during (blue), and $1 \mathrm{~s}$ following (red) light stimulation. Examples (left) are from 5 different animals. The example on the right demonstrates a 270 degree turn. The distance traveled during stimulation is reduced compared to before and after stimulation. G, Quantification of movement direction and speed relative to light onset. Photostimulation causes an abrupt shift in movement direction toward the ipsilateral side, accompanied by a reduction in locomotor speed. Data are mean \pm standard error mean, $n=5,3-9$ trials per animal. $\mathbf{H}$, Body center-to-head vectors are plotted as a function of time $0.6 \mathrm{~s}$ before (black), $1 \mathrm{~s}$ during (blue), and $0.6 \mathrm{~s}$ following (red) light stimulation.

turning, we found that $\mathrm{CNO}$ administration caused a strong preference in turning toward the side of ChxlO Gi activation (the ipsilateral side, Figure 3B). We next examined locomotion in an open field. CNO administration strongly induced ipsilateral turning even during unrestricted, spontaneous locomotion (Figure 3C, Video S1). CNO administration had no behavioral effect in control injected animals (Figure S2).

Quantification of the percentage of ipsilateral versus contralateral revolutions in open-field analysis revealed that ipsilateral turning developed over time (Figure 3D), approaching $100 \%$ ipsilateral turning preference by 10 minutes. At early stages following drug administration (5-15 minutes), these ipsilateral turns were smooth-similar to those observed during spontaneous changes in locomotor direction. There were no clear changes in trunk (axial) posture at early stages following administration of CNO. At late stages (> 20 min), we observed an ipsilateral axial bend which was evident even at rest (Video S1). Notably, during both early and late stages following administration of $\mathrm{CNO}$, bona fide turns - where locomotor direction actually changed-only occurred when the animal started moving forward, suggesting specific involvement of the limbs in imparting changes to locomotor direction.

To further investigate the dynamics of this behavior, we performed short-lasting photo-stimulation of Chx10 Gi neurons after unilateral expression of channelrhodopsin-2 $\left(C h \times 10^{C r e}>\right.$ AAV-FLEX-ChR2) (Figure 3E). Animals were stimulated with blue light $(473 \mathrm{~nm})$ for $1 \mathrm{~s}$ when slowly walking in an open field. Movement trajectories were calculated 1 s before, during, and after stimulation (Figure 3F). In all cases (27 trials in 5 animals), photostimulation led to an abrupt turn toward the ipsilateral side (Figure 3F, Video S2). This ipsilateral turn was initiated within $150 \mathrm{~ms}$ and lasted approximately $1.2 \mathrm{~s}$ (Figure $3 \mathrm{G}$ and $3 \mathrm{H}$ ), and was temporally cor- 
A

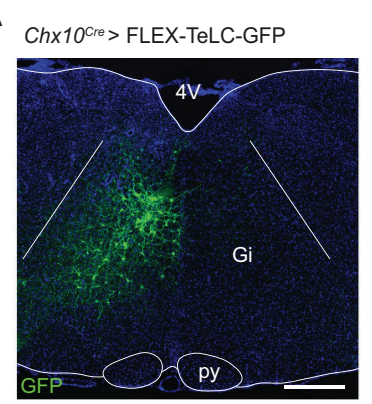

E

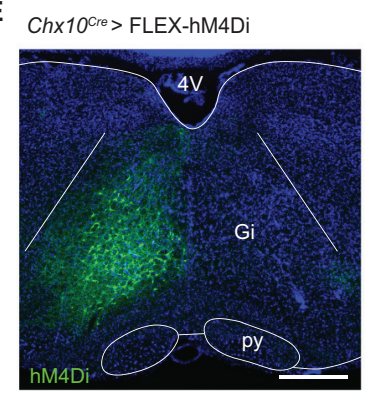

B

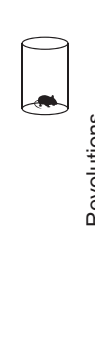

$\mathbf{F}$

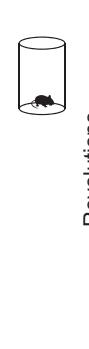

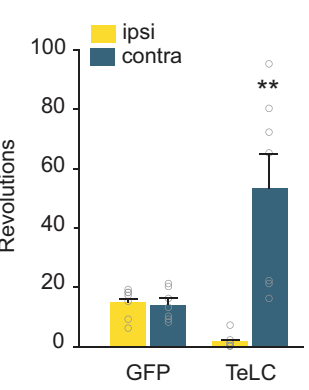

C

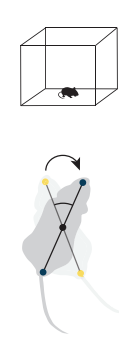

G

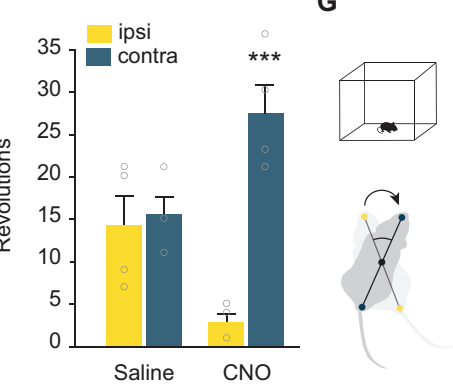

D

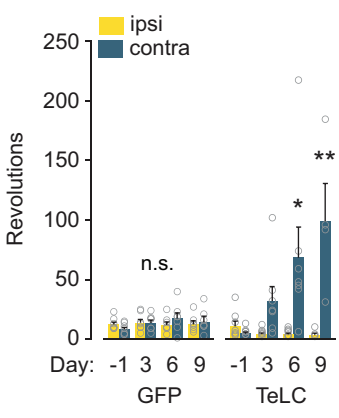

H

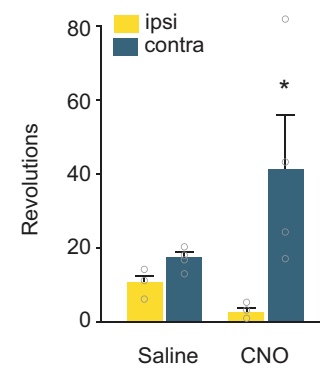

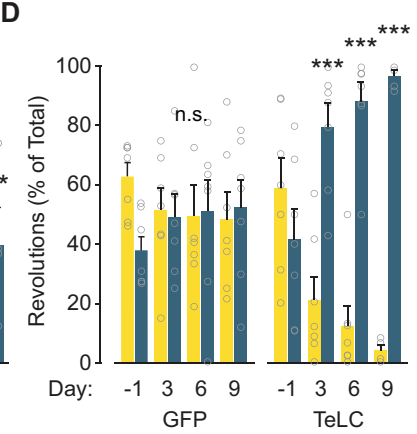

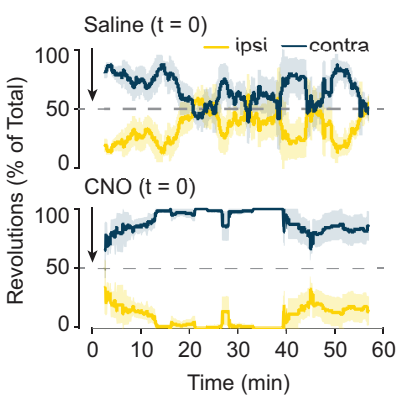

Figure 4. Inhibition of Chx10 Gi neurons causes contralateral movements. A, Unilateral injection of AAV-FLEX-TeLC-GFP or AAVFLEX-GFP in $C h \times 10^{C r e}$ mice. Example of GFP expression in Gi 2 weeks post-injection. Scale bar $=500 \mu \mathrm{m}$. B, Turning preference in a 10 minute cylinder assay $7 \mathrm{~d}$ after viral injection. ${ }^{* \star} P<0.0013$, one-way ANOVA with Tukey HSD, $n=7$. C, Turning preference in an open field assay, quantified as the total number of revolutions. GFP: $P>0.35$ for each comparison. TeLC: day $-1, P=0.99$ ipsi vs. contra; day $3, P=0.77$; day $6,{ }^{*} P=0.017$; day $9,{ }^{*} P=0.0048$. One-way ANOVA with Tukey HSD. D, Turning preference in an open field assay quantified as the percentage of total revolutions over a $30 \mathrm{~min}$ trial. GFP: $P>0.41$ for each comparison. TeLC: day $-1, P$ $=0.76$ ipsi vs. contra; day $3,{ }^{* * \star} P=9.7 \times 10^{-5}$; day $6,{ }^{* * *} P=5.9 \times 10^{-7}$; day $9,{ }^{* * *} P=3.2 \times 10^{-6}$. One-way ANOVA with Tukey HSD. E, Example of hM4Di expression after unilateral injection of AAV-FLEX-hM4Di in Chx $10^{C r e}$ mice. F, Movement preference in a 10 minute cylinder assay $20 \mathrm{~min}$ after injection of saline or CNO. ${ }^{* *} P=0.0002$, one-way ANOVA with Tukey HSD, $n=4$. G, Movement preference in an open field assay, quantified between 30-60 min post-injection of saline or CNO. ${ }^{*} P=0.012$, one-way ANOVA with Tukey HSD, $n$ $=4$. $\mathbf{H}$, Instantaneous analysis of movement preference, quantified as the percentage of ipsilateral versus contralateral revolutions (bin $=5 \mathrm{~min}$ ), following injection of saline (top) or $\mathrm{CNO}$ (bottom) at $\mathrm{t}=0$.

related with a reduction in locomotor speed (Figure $3 \mathrm{~F}$ and $3 \mathrm{G})$. Photostimulation at rest could not evoke a locomotor turn, and instead caused head and trunk bending toward the ipsilateral side (Video S2). In some cases, the animal would perform a 270-degree turn (Figure 3F). Such sharp turns were accompanied by bending of the trunk, suggesting involvement of the axial muscles at least for pronounced changes in direction.

These experiments indicate that Chxl0 Gi neurons evoke ipsilateral movements via two distinct mechanisms. Stimulation of Chx10 Gi neurons at rest evoked ipsilateral trunk/head movements, whereas stimulation during ongoing locomotion caused locomotor movements toward the ipsilateral side. Chxl0 Gi neurons appear to encompass all modes of turning; increasing Chxlo Gi activity caused changes in trunk posture at rest as well as either gradual and/or sharp changes in locomotor direction.

\section{Inhibition of Chx10 Gi neurons causes contralateral movements.}

We next examined the behavioral consequence of unilateral inactivation of ChxlO Gi neurons using a Cre-dependent tetanus toxin virus $\left(\mathrm{Ch} \times 10^{\mathrm{Cre}}>\mathrm{AAV}-\mathrm{FLEX}-\mathrm{TeLC}-\mathrm{GFP}\right)$
(Figure 4A). In the cylinder assay, we found that FLEXTeLC (but not FLEX-GFP) injected mice exhibited a strong turning preference toward the contralateral side (Figure 4B). We performed a time course analysis of turning preference after TeLC injection in the open field. One day before injection of FLEX-TeLC-GFP or FLEX-GFP, mice exhibited no open-field turning preference (Figures 4C and 4D, Video S3). Animals injected unilaterally with FLEX-TeLC (but not FLEX-GFP) started to exhibit turning contralateral to the injected side as soon as 3 days after injection (Figures $4 \mathrm{C}$ and 4D). By 9 days, $100 \%$ of TeLC animals exhibited contralateral turning (Figures 4C and 4D, Video S3). At days 3-6, contralateral turns were smooth, and there were no obvious effects on trunk posture when the animal was at rest (Video S3). When fully developed at day 9 , turns were sharper than in the early stages (Video S3). Therefore, similar to unilateral Chxlo Gi stimulation, inhibition of Chxl0 Gi activity caused both gradual and sharp modes of locomotor turning-but toward the contralateral side. We further confirmed these results by acute inactivation of Chxl0 Gi neurons using inhibitory DREADDS (Chx10 ${ }^{\text {Cre }}>$ FLEX-hM4Di), which also promoted contralateral movements (Figures 4E-4H). 


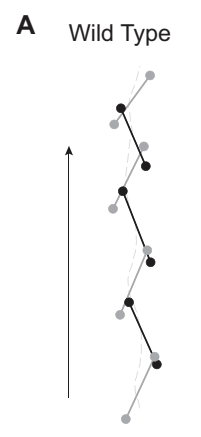

straight

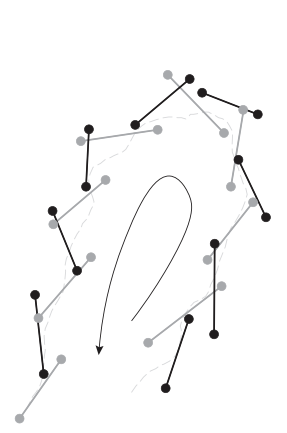

left turn
B

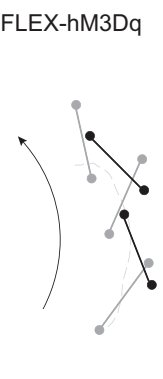

ipsilateral turn
C FLEX-TeLC

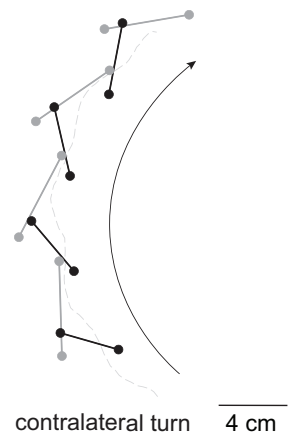

D

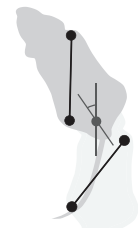

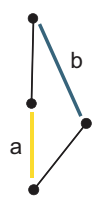

E

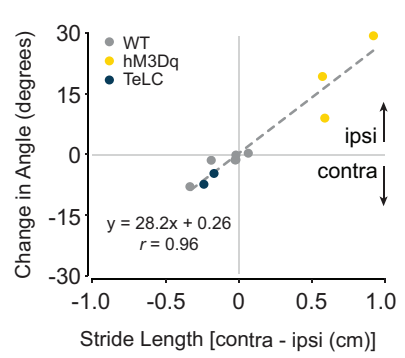

$\mathbf{F}$

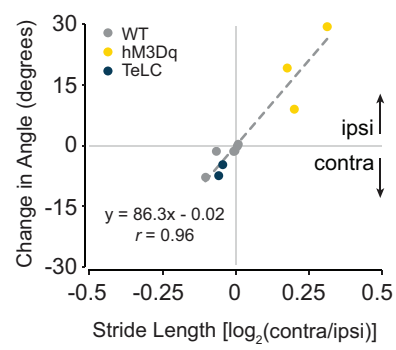

Figure 5. Limb dynamics during natural and Chx10-induced turns. A, Examples of footfalls in a wild-type mouse walking straight and making a spontaneous left turn. Black lines indicate the left-forelimb/right-hindlimb diagonal, whereas gray lines indicate the rightforelimb/left-hindlimb diagonal. For spontaneous turns, the limb on the side of the turn exhibits shorter steps than its corresponding diagonal. B, Example of footfalls in a left Gi $\mathrm{Ch} \times 10^{\mathrm{Cre}}>\mathrm{FLEX}-\mathrm{hM} 3 \mathrm{Dq}$ mouse after injection of CNO, which causes turning toward the ipsilateral (left) side. Steps are shorter on the ipsilateral side. C, Example of footfalls in a left Gi Chx10 Cre $>$ FLEX-TeLC mouse, which causes turning toward the contralateral (right) side. Steps are longer on the ipsilateral side. D, Schematic of turning based on data in A-C. A single step can exhibit left/right motor asymmetry, where the ipsilateral hindlimb exhibits a shorter stride length (a) than the diagonal limb of the contralateral side (b) leading to changes in heading position. E-F Mathematical models demonstrating that a difference in stride length predicts a difference in heading position. We quantified footfalls in wild-type, Chx $10^{C r e}>$ FLEX-hM3Dq, and $\mathrm{Ch} \times 10^{\mathrm{Cre}}>\mathrm{FLEX}$-TeLC mice. For wild-type mice, the difference in stride length, measured either as the absolute distance (E) or as the $\log _{2}$ ratio $(F)$, hovered around zero, which corresponds to straight walking and/or slight turning to one side. For FLEX-hM3Dq mice after CNO injection, the values are positive-predicting an ipsilateral turn. For FLEX-TeLC mice, the values are negative-predicting a contralateral turn.

Unilateral inactivation of Chx10 Gi neurons dramatically increased the total distance animals moved during a $30 \mathrm{~min}$ probe (Figure S3). These data suggest that Chx10 Gi neurons act as a unilateral 'brake' on locomotion, and removing this unilateral brake dramatically increases locomotor speed.

\section{Limb dynamics during natural and drug-evoked turns.}

Our data strongly suggest that the mechanism for Chxl0evoked locomotor turns reflects distinct changes in limb dynamics: ChxlO-evoked ipsilateral turns were accompanied by a decrease in locomotor speed (Figure $3 \mathrm{~F}$ and $3 \mathrm{G}$ ), whereas inhibition of Chx10 Gi neurons caused contralateral turning accompanied by an increase in overall locomotor speed (Figure S3).

To investigate these limb dynamics in greater detail, we analyzed how limb coordination relates to locomotor direction in freely moving mice. DeepLabCut was used for markerless extraction of forelimb and hindlimb paw position in an open field (Video S4) (45). We focused on continuous locomotor bouts with a speed $>15 \mathrm{~cm} \mathrm{~s}^{-1}$, which corresponds to fast walk or trot (46). For locomotor bouts in wild-type animals, locomotion that proceeded in a straight line exhibited a similar stride length on the left and right sides (Figure 5A). During spontaneous turns, stride length was reduced on the side of the turn (Figure 5A). We compared spontaneous turns in wild-type mice with those biased to one side via excitation (Chx10 Cre $>$ hM3Dq-DREADDs after CNO) or inhibition (Chx10 Cre $>$ FLEX-TeLC) of Chxl0 Gi neurons (Figures 5B and 5C). Turns in wild-type animals and those caused by $\mathrm{Ch} \times 10^{\mathrm{Cre}}>$ FLEX-hM3Dq or Chx10 Cre $>$ FLEXTeLC shared a defining characteristic, that is, the hindlimbs and the forelimbs on the side of the turn travelled a shorter distance than the leg opposite to the turn (Figures 5B and $5 \mathrm{C})$.

We next asked whether differences in stride length between the left and right sides were sufficient to predict locomotor direction (Figure 5D). We calculated the relationship between the distance travelled by the right and left limbs with heading direction for all locomotor bouts sampled in wildtype, Chx $10^{\mathrm{Cre}}>$ FLEX-hM3Dq, and Chx $10^{\mathrm{Cre}}>$ FLEXTeLC mice. In wild-type mice, we observed epochs with no difference in left/right stride length, which corresponded to straight walking, as well as epochs with longer left leg 

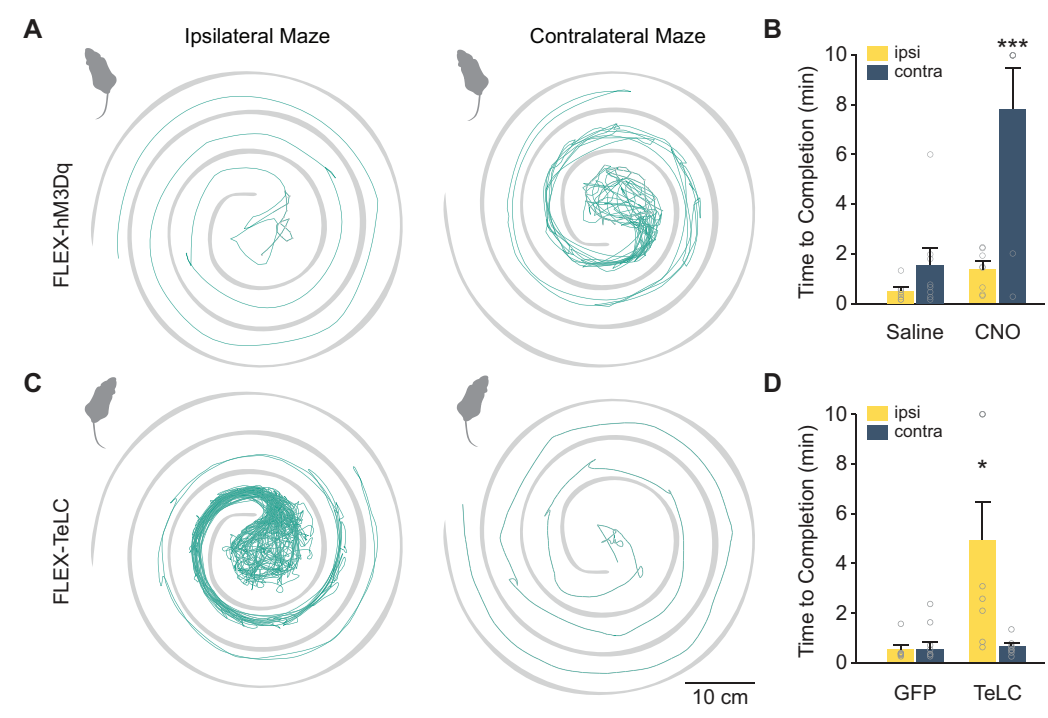

D

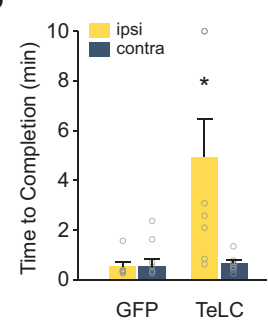

Figure 6. Unilateral function of Chx10 Gi neurons is critical for left/right movements during natural exploratory behaviors. A, Activation of Chx10 Gi neurons unilaterally with excitatory hM3Dq-DREADDs impairs exploration in a contralateral (right-turn) maze. B, 6 of 8 CNO injected animals failed to complete the contralateral maze during a 10 min trial. ${ }^{\star * \star} P<5 \times 10^{-5}$, one-way ANOVA with Tukey HSD, $n=8$. C, Inhibition of Chx10 Gi neurons unilaterally with TeLC impairs exploration in an ipsilateral (left-turn) maze. D, 3 of 8 TeLC injected animals failed to complete the ipsilateral maze during a 10 min trial. ${ }^{*} P<0.011$, one-way ANOVA with Tukey HSD, $n=$ 7 for GFP and $n=8$ for TeLC.

steps (turning right) and longer right leg steps (turning left). Chx $10^{\text {Cre }}>$ FLEX-hM3Dq mice exhibited shorter steps on the side of injection, which caused ipsilateral turns. Chx $10^{\mathrm{Cre}}$ $>$ FLEX-TeLC mice exhibited longer steps on the side of injection, which caused contralateral turns (Figures 5E and 5F). For each animal, we observed a positive correlation between the difference in stride length and heading direction $(r=0.34$ 0.53). Together these data lead to a model where the difference in stride length on the left and right sides predicts locomotor direction $(r=0.96$ for absolute distance, Figure 5E; $r$ $=0.96$ for $\log _{2}$ ratio, Figure $5 \mathrm{~F}$ ). These data are compatible with the hypothesis that increased $C h x 10 \mathrm{Gi}$ activity reduces locomotor rhythmogenic activity (9) on the ipsilateral side, leading to shorter steps on the side of the turn.

\section{Chx10 Gi neurons function to control left/right move- ments during natural exploratory behaviors.}

To test the necessity/sufficiency of Chx10 Gi neurons for turns during natural exploratory behaviors, we designed a paradigm where animals explored a novel left- or right-turned maze (Figure 6A). Animals were placed in the center of this simple spiral-shaped maze, which they explored until they exited the maze or until 10 minutes had elapsed. Unaffected mice rapidly completed both left- and right-turned mazes (Figures 6A-6D). In contrast, unilateral activation of Chx10 stop neurons with hM3Dq-DREADDs increased exploratory time in a contralateral but not ipsilateral maze (Figures 6A and $6 \mathrm{~B}$ ), with several mice failing to complete the contralateral maze altogether $(n=6 / 8$, Figure $6 \mathrm{~B})$. Mirroring this effect, inactivation of ChxlO stop neurons with TeLC increased exploratory time in an ipsilateral but not contralateral maze (Figure 6C), with several mice failing to complete the ipsilateral maze $(n=3 / 8$, Figure $6 \mathrm{D})$. These experiments show that animals do not have an ability to compensate for dysfunction of the ChxlO turning system, which appears to be required for natural exploratory function.

\section{Neurons of the superior colliculus provide a unilateral input that links Chx10 Gi neurons to natural left/right movements.}

These experiments define a final command pathway for executing left/right locomotor asymmetries. The remaining question is whether it is possible to recruit ChxlO Gi neurons unilaterally during natural behaviors? To address this question, we first performed monosynaptically restricted transsynaptic labeling from $\mathrm{Ch} x \mathrm{lO}$ Gi neurons to determine their immediate synaptic inputs $(47,48)$. Cre-dependent rabies helper virus (AAV-FLEX-helper, see methods) was injected in the left $\mathrm{Gi}$ of $C h \times 10^{\mathrm{Cre}}$ mice, followed by RV $\Delta \mathrm{G}$ $4 \mathrm{mCherry}($ EnvA). Consistent with our results using anterograde tracing (Figure 1B), monosynaptic retrograde tracing demonstrated that Chx10 Gi neurons do not exhibit strong connectivity across the midline (Figures S4B and S4C); Chx10 Gi neuronal populations are not reciprocally connected. Chx10 Gi neurons received several long-range inputs that were primarily unilateral from the contralateral superior colliculus (SC), the ipsilateral zona incerta, the ipsilateral mesencephalic reticular formation $(\mathrm{mRt})$ and the contralateral medial cerebellar (the fastigial) nucleus (Figures 7A and S4, Table S1). Chx10 Gi neurons also exhibited bilateral long-range inputs from the lateral cerebellar (the dentate) nuclei and sensorimotor cortex (Figure S4, Table S1). Notably, no transsynaptic labeling was observed from the cuneiform or pedunculopontine nuclei, which comprise the midbrain locomotor region (Table S1). These experiments provide anatom- 

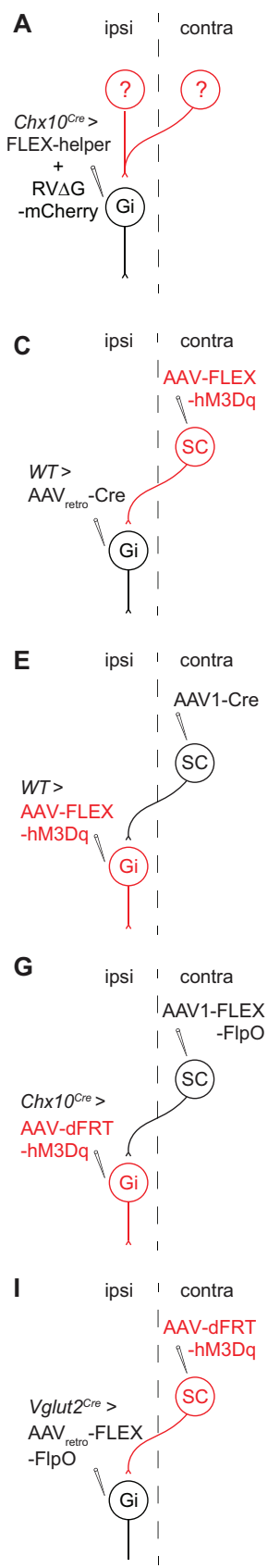
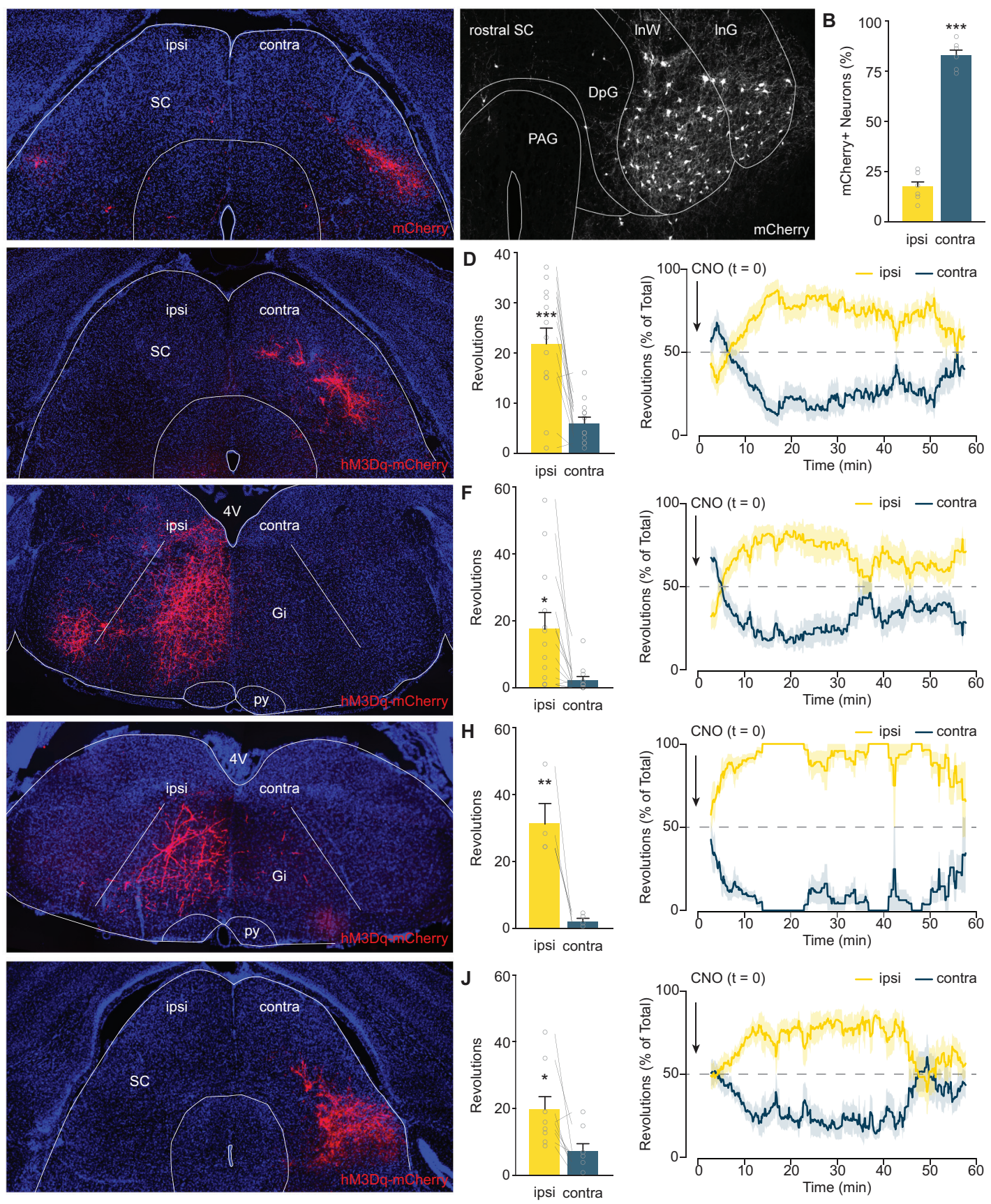

Figure 7. Contralateral SC steering acts through $\mathrm{Chx10}$ Gi neurons. A, A rabies transsynaptic tracing approach was used to identify presynaptic inputs to Chx10 Gi neurons (see also, Figure S4 and Table S1). Rabies-mCherry tracing revealed a prominent input from the contralateral SC, where neurons occupied intermediate layers (left, caudal SC; right, rostral SC). B, Quantification of mCherry-labeled neurons in the ipsilateral and contralateral SC. ${ }^{* *} P=1.8 \times 10^{-8}$, two-tailed t-test, $n=6$. C, Retrograde behavioral interrogation of the SC-Gi synapse in wild type mice. Injection of $A A V_{\text {retro }}-$ Cre in the Gi caused recombination of a FLEX-hM3DqmCherry virus injected in $\mathrm{SC}_{\text {contra. }}$ D, CNO injection caused an ipsilateral turning preference, ${ }^{\star \star \star} P=9.2 \times 10^{-5}$, two-tailed t-test, $n=$ 13. Right, Instantaneous quantification of turning percentage after CNO injection. E, Anterograde behavioral interrogation of the SC-Gi synapse in wild type mice. Injection of AAV1-Cre in $\mathrm{SC}_{\text {contra }}$ caused recombination of a FLEX-hM3Dq-mCherry virus injected in Gi $\mathrm{i}_{\text {ipsi }}$. $\mathrm{F}, \mathrm{CNO}$ injection caused an ipsilateral turning preference. ${ }^{\star} P=0.011$, two-tailed t-test, $n=13$. Right, Instantaneous quantification of turning percentage after CNO injection. G, Anterograde behavioral interrogation of the SC-Gi synapse in $\mathrm{Ch} \times 10^{C r e}$ mice. Injection of AAV1-FLEX-FlpO in $\mathrm{SC}_{\text {contra }}$ caused recombination of a dFRT-hM3Dq-mCherry virus injected in $\mathrm{Gi}_{i p s i}$. $\mathrm{H}$, CNO injection caused an ipsilateral turning preference. ${ }^{* \star} P=0.003$, two-tailed t-test, $n=4$. Right, Instantaneous quantification of turning percentage after

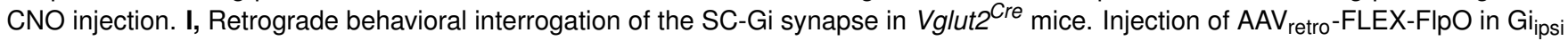
caused recombination of a dFRT-hM3Dq-mCherry virus injected in $\mathrm{SC}_{\text {contra. }} \mathrm{J}, \mathrm{CNO}$ injection caused an ipsilateral turning preference. ${ }^{*} P=0.016$, two-tailed t-test, $n=9$. Right, Instantaneous quantification of turning percentage after CNO injection. 
ical evidence that activity of $C h x 10$ Gi neurons can be biased via unilateral input from upstream nuclei.

To test this possibility we stimulated the SC-Gi projection. We focused on the SC because of its prominent role in sensorimotor integration (49-51), and for its role in gaze and head orientation within 3D space (52). Projections from the contralateral SC to Gi have been described (51). We now show that these SC-Gi projection neurons occupy intermediate layers of the SC, and they synapse specifically with Chx10 Gi neurons (Figures 7A and 7B). We tested the behavioral significance of this SC-Gi projection in four different ways. First, the SC-Gi projection was targeted by retrograde labeling of $\mathrm{SC}$ neurons using $\mathrm{Gi}_{\text {ipsi }}>\mathrm{AAV}_{\text {retro }}-\mathrm{Cre}$ followed by $\mathrm{SC}_{\text {contra }}>$ FLEX-hM3Dq (Figure 7C). SC neurons targeted using this approach had a discrete laminar position which matched the rabies transsynaptic tracing (Figures 7A and 7C). In these mice, CNO administration rapidly initiated ipsilateral turning (Figure 7D). Next, postsynaptic Gi neurons were targeted using $\mathrm{SC}_{\text {contra }}>\mathrm{AAV} 1-\mathrm{Cre}$, an anterograde transsynaptic virus (51), followed by $\mathrm{Gi}_{i p s i}>$ FLEXhM3Dq (Figure 7E). Gi neurons exhibited robust recombination on the ipsilateral side (Figure 7E). Again, CNO administration rapidly initiated ipsilateral turning (Figure 7F).

We supplemented these approaches using an intersectional strategy to allow both projection- and target-specific recombination in postsynaptic neurons (Figures 7G-J). In Chx $10^{\text {Cre }}$ mice, targeting postsynaptic $\mathrm{Gi}$ neurons using $\mathrm{SC}_{\text {contra }}>$ AAV1-FLEX-FlpO followed by $\mathrm{Gi}_{\text {ipsi }}>\mathrm{dFRT}-$ hM3Dq caused recombination in ipsilateral Chx10 Gi neurons (Figure 7G). CNO administration rapidly initiated ipsilateral turning (Figures 7H). Finally, we showed that SCGi projection neurons are glutamatergic. In Vglut $2^{\text {Cre }}$ mice (53), glutamatergic SC-Gi projection neurons were targeted using $\mathrm{Gi}_{\text {ipsi }}>\mathrm{AAV}_{\text {retro }}-$ FLEX-FlpO followed by $\mathrm{SC}_{\text {contra }}>$ dFRT-hM3Dq (Figure 7I). CNO administration rapidly initiated ipsilateral turning (Figure $7 \mathrm{~J}$ ). These results show that a natural sensorimotor pathway can bias Chxlo Gi neuronal activity during spontaneous locomotion. Moreover, these experiments unambiguously demonstrate that the SC imparts left/right locomotor directional commands via Chx10 Gi neurons.

\section{DISCUSSION}

The present study uncovers a command system that enables left/right locomotor asymmetries necessary for directional movements. This asymmetric reticulospinal command system functions in parallel with symmetric start and speed control circuits $(10,11,14,18)$, and may be used symmetrically to arrest ongoing locomotion (9). Our study, therefore, completes the description of the three main requisite control components for locomotion: start, stop, and direction. Remarkably, when Chxlo Gi neurons were biased to produce either ipsilateral or contralateral movements, animals could not perform a compensatory turn. This observation suggests that the turning system revealed here is the dominant system used during natural behaviors, and is recruited by brain circuits involved in meditating directional movements.

\section{Mechanism for locomotor turns.}

In aquatic vertebrates, left/right steering movements are achieved by asymmetric activity of glutamatergic reticulospinal neurons on the left and the right sides. This causes a forceful contraction of axial muscles on the side of the turn (54-59). In our experiments, we show that asymmetric movements in mice are also caused by an imbalance in descending excitation between the left and right sides. Nonetheless, the limb-based mechanism for turning is opposite to that described in species which use axial muscles as the predominant locomotor effector: Unilateral activation of mammalian Chx10 Gi neurons initiates ipsilateral turning, which is caused by a reduction of locomotor speed and stride length on the ipsilateral side. Thus, for the limbs, excitation in spinal limb locomotor circuits is reduced on the side of the turn. In previous studies we showed that bilateral activation of Chx 10 Gi neurons arrests or reduces limbed locomotor activity via a brake on locomotor rhythm generation in the cord (9). The unilateral Chx10 Gi gain- and loss-of-function experiments demonstrate that a unilateral brake (activation of ChxlO Gi neurons) or release of the brake (inactivation of $C h x 10 \mathrm{Gi}$ neurons) is sufficient to mediate a limb-based turn-where speed and stride length are higher on the side opposite to the turn.

We suggest that this turning mechanism for limbed species evolved to account for general features of the limbed body plan. Chiefly, axial muscles generate force perpendicular to the directional axis. In contrast, limbs generate force parallel to the directional axis but at a distance from the midline, creating a moment about the medial axis. Although locomotor direction is ultimately mediated by the limbs and requires locomotion (60), unilateral stimulation of Chxlo Gi neurons evoked bending of the head and trunk toward the side of stimulation even at rest. Based on these observations we propose that limbed animals use a two-component system for directing movements to the left or right side. Perhaps not surprisingly, this two-component biological turning system is a design principle adopted for steering four-wheeled vehicles millions of years after it was selected during evolution to control left/right locomotor asymmetries in quadrupeds: turning in quadrupeds and four-wheeled vehicles is enabled by a dedicated steering/differential system for independent control of speed on the left and right sides.

\section{Integrated function of $\operatorname{Chx} 10$ Gi neurons.}

Mammalian Chxlo Gi neurons were initially associated with locomotor stop, a behavioral response caused by bilateral activation (9). The data presented here give clear evidence that asymmetric engagement of Chx10 Gi neurons causes turning. Chx10 Gi neurons may therefore have a dual function-stop or turn-dependent on their symmetric or asymmetric activation. The basis for differential engagement may rest in task-dependent recruitment from upstream 
brain areas. Accordingly, our rabies tracing screen demonstrated that certain presynaptic nuclei contribute prominent bilateral or unilateral input. Prominent bilateral inputs from the lateral deep cerebellar nuclei and sensorimotor cortex (among others) provide an anatomical basis for a symmetric stop command. Prominent unilateral input from glutamatergic neurons in the intermediate area of the contralateral superior colliculus, the ipsilateral zona incerta, the ipsilateral $\mathrm{mRt}$, and the contralateral medial deep cerebellar (fastigial) nucleus provide a basis for recruiting an asymmetric turn command. Indeed, we demonstrate that activation of the crossed SC-Chx10 Gi pathway mediates movements contralateral to the superior colliculus. These experiments clearly demonstrate that $C h x 10 \mathrm{Gi}$ neurons can be recruited to generate locomotor asymmetries through the superior colliculus, a hub for sensorimotor integration $(51,52)$.

Interestingly, the superior colliculus, zona incerta, and $\mathrm{mRt}$ were recently identified in a brain-wide screen for neurons which control the decision to move left or right (31). Further, it has been shown that glutamatergic fastigial neurons project to the contralateral Gi (61). The present brain-wide screen links this excitatory cerebellar output to Chx10 Gi neurons. Fastigial neurons are downstream of the vestibule-cerebellum, which has been shown to encode turning during locomotion (62). Glutamatergic inputs from contralateral SC and fastigial neurons to $C h x 10$ Gi neurons, therefore, contribute information from both sensorimotor and vestibular sources, information which ultimately biases locomotor movements to the ipsilateral side.

Together our findings suggest that Chx10 Gi neurons act as the final common effector for left/right movements, and might act as a locus for integration of diverse sensory signals which contribute to the decision to move left or right. Understanding the specific contribution of brain areas upstream of Chx10 Gi neurons-and how they work in concert-is expected to lead to a more thorough understanding of how motor control is organized at the behavioral level.

Acknowledgments. We thank K. Sharma, L. Zagoraiou, S. Crone, and T.M. Jessell for the Chx10 Cre mouse. We acknowledge the Core Facility for Integrated Microscopy, Faculty of Health and Medical Sciences, University of Copenhagen. We thank Iryna Vesth-Hansen, Dorthe Meinertz, and Paulina Wanken for technical assistance, and members of Ole Kiehn's lab for discussion and comments on previous versions of this manuscript. This work was supported by an EMBO Long-Term Fellowship (J.M.C., ALTF 421-2018), a European Research Council Advanced Grant (O.K., LocomotorIntegration, REP-SCI-693038), and the Novo Nordisk Laureate Program (O.K., NNF15OC0014186).

Author contributions. Conceptualization, J.M.C. and O.K.; Methodology, J.M.C., R.L., A.M., and O.K.; Investigation, J.M.C., R.L., A.M.; Resources - I.R.W.; Writing - Original Draft, J.M.C., O.K.; Writing - Review \& Editing, J.M.C., R.L., and O.K.; Supervision - O.K.; Funding Acquisition, J.M.C., O.K.
Competing interests. The authors declare no competing interests.

\section{REFERENCES}

1. Silvia Arber. Motor circuits in action: Specification, connectivity, and function. Neuron, 74(6):975-989, Jun 2012. ISSN 08966273. doi: 10.1016/j.neuron.2012.05.011.

2. Robert M Brownstone and Jennifer M Wilson. Strategies for delineating spinal locomotor rhythm-generating networks and the possible role of hb9 interneurones in rhythmogenesis. Brain research reviews, 57(1):64-76, Jan 2008. ISSN 0165-0173. doi: 10.1016/j. brainresrev.2007.06.025.

3. Martyn Goulding. Circuits controlling vertebrate locomotion: moving in a new direction. Nature Reviews Neuroscience, 10(7):507-518, Jul 2009. ISSN 1471-003X. doi: 10.1038/nrn2608.

4. Sten Grillner. The motor infrastructure: from ion channels to neuronal networks. Nature Reviews Neuroscience, 4(7):573-586, Jul 2003. ISSN 1471-003X. doi: 10.1038/nrn1137.

5. Sten Grillner and Thomas M Jessell. Measured motion: searching for simplicity in spinal locomotor networks. Current Opinion in Neurobiology, 19(6):572-586, Dec 2009. ISSN 09594388. doi: 10.1016/j.conb.2009.10.011.

6. Ole Kiehn. Locomotor circuits in the mammalian spinal cord. Annual Review of Neuroscience, 29(1):279-306, Jul 2006. ISSN 0147006X. doi: 10.1146/annurev.neuro.29.051605.112910.

7. Ole Kiehn. Decoding the organization of spinal circuits that control locomotion. Nature Reviews Neuroscience, 17(4):224-238, Mar 2016. ISSN 1471-003X. doi: 10.1038/nrn.2016.9.

8. D M Armstrong. Supraspinal contributions to the initiation and control of locomotion in the cat. Progress in neurobiology, 26(4): 273-361, 1986. ISSN 0301-0082.

9. Julien Bouvier, Vittorio Caggiano, Roberto Leiras, Vanessa Caldeira, Carmelo Bellardita, Kira Balueva, Andrea Fuchs, and Ole Kiehn. Descending command neurons in the brainstem that halt locomotion. Cell, 163(5):1191-1203, Nov 2015. ISSN 00928674. doi: 10.1016/j.cell.2015.10.074.

10. V. Caggiano, R. Leiras, H. Goñi-Erro, D. Masini, C. Bellardita, J. Bouvier, V. Caldeira, G. Fisone, and O. Kiehn. Midbrain circuits that set locomotor speed and gait selection. Nature, 553(7689): 455-460, Jan 2018. ISSN 0028-0836. doi: 10.1038/nature25448.

11. Paolo Capelli, Chiara Pivetta, Maria Soledad Esposito, and Silvia Arber. Locomotor speed control circuits in the caudal brainstem. Nature, 551(7680):373-377, Oct 2017. ISSN 0028-0836. doi: 10. 1038/nature24064.

12. S Grillner, Ekeberg, A El Manira, A Lansner, D Parker, J Tegnér, and $P$ Wallén. Intrinsic function of a neuronal network - a vertebrate central pattern generator. Brain research. Brain research reviews, 26(2-3):184-97, May 1998.

13. Larry M. Jordan, Jun Liu, Peter B. Hedlund, Turgay Akay, and Keir G. Pearson. Descending command systems for the initiation of locomotion in mammals. Brain Research Reviews, 57(1):183-191, Jan 2008. ISSN 01650173. doi: 10.1016/j.brainresrev.2007.07.019.

14. Nicolas Josset, Marie Roussel, Maxime Lemieux, David LafranceZoubga, Ali Rastqar, and Frederic Bretzner. Distinct contributions of mesencephalic locomotor region nuclei to locomotor control in the freely behaving mouse. Current Biology, 28(6):884-901.e3, Mar 2018. ISSN 09609822. doi: 10.1016/j.cub.2018.02.007.

15. Laurent Juvin, Swantje Grätsch, Emilie Trillaud-Doppia, JeanFrançois Gariépy, Ansgar Büschges, and Réjean Dubuc. A specific population of reticulospinal neurons controls the termination of locomotion. Cell Reports, 15(11):2377-2386, Jun 2016. ISSN 22111247. doi: 10.1016/j.celrep.2016.05.029.

16. Jun Liu and Larry M Jordan. Stimulation of the parapyramidal region of the neonatal rat brain stem produces locomotor-like activity involving spinal 5-ht7 and 5-ht2a receptors. Journal of neurophysiology, 94(2):1392-404, Aug 2005. ISSN 0022-3077. doi: 10.1152/jn.00136.2005. 
bioRxiv preprint doi: https://doi.org/10.1101/754812; this version posted September 3, 2019. The copyright holder for this preprint (which was not certified by peer review) is the author/funder, who has granted bioRxiv a license to display the preprint in perpetuity. It is made available under aCC-BY-ND 4.0 International license.

17. P. E. Musienko, P. V. Zelenin, V. F. Lyalka, Y. P. Gerasimenko, G. N. Orlovsky, and T. G. Deliagina. Spinal and supraspinal control of the direction of stepping during locomotion. Journal of Neuroscience, 32(48):17442-17453, Nov 2012. ISSN 0270-6474. doi: 10.1523/ JNEUROSCI.3757-12.2012.

18. Thomas K. Roseberry, A. Moses Lee, Arnaud L. Lalive, Linda Wilbrecht, Antonello Bonci, and Anatol C. Kreitzer. Cell-typespecific control of brainstem locomotor circuits by basal ganglia. Cell, 164(3):526-537, Jan 2016. ISSN 00928674. doi: 10.1016/ j.cell.2015.12.037.

19. Dimitri Ryczko and Réjean Dubuc. The multifunctional mesencephalic locomotor region. Current pharmaceutical design, 19(24): 4448-70, 2013. ISSN 1873-4286.

20. M L Shik, F V Severin, and G N Orlovskiĭ. Control of walking and running by means of electric stimulation of the midbrain. Biofizika, 11(4):659-66, 1966.

21. Frédéric Brocard, Dimitri Ryczko, Karine Fénelon, Raja Hatem, Delphine Gonzales, François Auclair, and Réjean Dubuc. The transformation of a unilateral locomotor command into a symmetrical bilateral activation in the brainstem. The Journal of neuroscience: the official journal of the Society for Neuroscience, 30(2):523-33, Jan 2010. ISSN 1529-2401. doi: 10.1523/JNEUROSCI.3433-09.2010.

22. P.E. Musienko, P.V. Zelenin, V.F. Lyalka, G.N. Orlovsky, and T.G. Deliagina. Postural performance in decerebrated rabbit. Behavioural Brain Research, 190(1):124-134, Jun 2008. ISSN 01664328. doi: 10.1016/j.bbr.2008.02.011.

23. Dimitri Ryczko, Jackson J. Cone, Michael H. Alpert, Laurent Goetz, François Auclair, Catherine Dubé, Martin Parent, Mitchell F. Roitman, Simon Alford, and Réjean Dubuc. A descending dopamine pathway conserved from basal vertebrates to mammals. Proceedings of the National Academy of Sciences, 113(17):E2440-E2449, Apr 2016. ISSN 0027-8424. doi: 10.1073/pnas.1600684113.

24. Lukas C Bachmann, Alina Matis, Nicolas T Lindau, Petra Felder, Miriam Gullo, and Martin E Schwab. Deep brain stimulation of the midbrain locomotor region improves paretic hindlimb function after spinal cord injury in rats. Science translational medicine, 5(208): 208ra146, Oct 2013. ISSN 1946-6242. doi: 10.1126/scitranslmed. 3005972.

25. E Garcia-Rill and R D Skinner. The mesencephalic locomotor region. i. activation of a medullary projection site. Brain research, 411 (1):1-12, May 1987. ISSN 0006-8993.

26. E Garcia-Rill and R D Skinner. The mesencephalic locomotor region. ii. projections to reticulospinal neurons. Brain research, 411 (1):13-20, May 1987. ISSN 0006-8993.

27. $S$ J Shefchyk, R M Jell, and L M Jordan. Reversible cooling of the brainstem reveals areas required for mesencephalic locomotor region evoked treadmill locomotion. Experimental brain research, 56(2):257-62, 1984. ISSN 0014-4819.

28. J D Steeves and L M Jordan. Localization of a descending pathway in the spinal cord which is necessary for controlled treadmill locomotion. Neuroscience letters, 20(3):283-8, Dec 1980. ISSN 0304-3940.

29. J D Steeves and L M Jordan. Autoradiographic demonstration of the projections from the mesencephalic locomotor region. Brain research, 307(1-2):263-76, Jul 1984. ISSN 0006-8993.

30. Manuel J. Ferreira-Pinto, Ludwig Ruder, Paolo Capelli, and Silvia Arber. Connecting circuits for supraspinal control of locomotion. Neuron, 100(2):361-374, Oct 2018. ISSN 08966273. doi: 10.1016/j.neuron.2018.09.015.

31. Nicholas A. Steinmetz, Peter Zatka-Haas, Matteo Carandini, and Kenneth D. Harris. Distributed correlates of visually-guided behavior across the mouse brain. bioRxiv, page 474437, Nov 2018. doi: $10.1101 / 474437$.

32. Agnès Bonnot, Patrick J Whelan, George Z Mentis, and Michael J O'Donovan. Locomotor-like activity generated by the neonatal mouse spinal cord. Brain research. Brain research reviews, 40(1-3): 141-51, Oct 2002.

33. E Bracci, L Ballerini, and A Nistri. Localization of rhythmogenic networks responsible for spontaneous bursts induced by strychnine and bicuculline in the rat isolated spinal cord. The Journal of neuroscience: the official journal of the Society for Neuroscience, 16: 7063-7076, Nov 1996. ISSN 0270-6474.

34. M. Hagglund, K. J. Dougherty, L. Borgius, S. Itohara, T. Iwasato, and $\mathrm{O}$. Kiehn. Optogenetic dissection reveals multiple rhythmogenic modules underlying locomotion. Proceedings of the National Academy of Sciences, 110:11589-11594, Jul 2013. ISSN 00278424. doi: 10.1073/pnas.1304365110.

35. M Kato. Motoneuronal activity of cat lumbar spinal cord following separation from descending or contralateral impulses. Central nervous system trauma: journal of the American Paralysis Association, 4(4):239-48, 1987. ISSN 0737-5999.

36. O Kjaerulff and O Kiehn. Distribution of networks generating and coordinating locomotor activity in the neonatal rat spinal cord in vitro: a lesion study. The Journal of neuroscience: the official journal of the Society for Neuroscience, 16:5777-5794, Sep 1996. ISSN 0270-6474.

37. E. Kremer and A. Lev-Tov. Localization of the spinal network associated with generation of hindlimb locomotion in the neonatal rat and organization of its transverse coupling system. Journal of Neurophysiology, 77(3):1155-1170, Mar 1997. ISSN 0022-3077. doi: 10.1152/jn.1997.77.3.1155.

38. B R Noga, S J Shefchyk, J Jamal, and L M Jordan. The role of renshaw cells in locomotion: antagonism of their excitation from motor axon collaterals with intravenous mecamylamine. Experimental brain research, 66(1):99-105, 1987. ISSN 0014-4819.

39. Duo Jin, Yuanyuan Liu, Fang Sun, Xuhua Wang, Xuefeng Liu, and Zhigang $\mathrm{He}$. Restoration of skilled locomotion by sprouting corticospinal axons induced by co-deletion of pten and socs3. $\mathrm{Na}$ ture Communications, 6(1):8074, Dec 2015. ISSN 2041-1723. doi: 10.1038/ncomms9074.

40. G D Muir and I Q Whishaw. Complete locomotor recovery following corticospinal tract lesions: measurement of ground reaction forces during overground locomotion in rats. Behavioural brain research, 103(1):45-53, Aug 1999. ISSN 0166-4328.

41. T G Deliagina, L B Popova, and G Grant. The role of tonic vestibular input for postural control in rats. Archives italiennes de biologie, 135 (3):239-61, Jun 1997. ISSN 0003-9829.

42. Martin Hägglund, Lotta Borgius, Kimberly J Dougherty, and Ole Kiehn. Activation of groups of excitatory neurons in the mammalian spinal cord or hindbrain evokes locomotion. Nature Neuroscience, 13:246-252, Feb 2010. ISSN 1097-6256. doi: 10.1038/nn.2482.

43. O Kjaerulff and O Kiehn. Crossed rhythmic synaptic input to motoneurons during selective activation of the contralateral spinal locomotor network. The Journal of neuroscience: the official journal of the Society for Neuroscience, 17(24):9433-47, Dec 1997. ISSN 0270-6474.

44. B. N. Armbruster, X. Li, M. H. Pausch, S. Herlitze, and B. L. Roth. Evolving the lock to fit the key to create a family of $g$ protein-coupled receptors potently activated by an inert ligand. Proceedings of the National Academy of Sciences, 104(12):5163-5168, Mar 2007. ISSN 0027-8424. doi: 10.1073/pnas.0700293104.

45. Alexander Mathis, Pranav Mamidanna, Kevin M. Cury, Taiga Abe, Venkatesh N. Murthy, Mackenzie Weygandt Mathis, and Matthias Bethge. Deeplabcut: markerless pose estimation of userdefined body parts with deep learning. Nature Neuroscience, 21(9):1281-1289, Sep 2018. ISSN 1097-6256. doi: 10.1038/ s41593-018-0209-y.

46. Carmelo Bellardita and Ole Kiehn. Phenotypic characterization of speed-associated gait changes in mice reveals modular organization of locomotor networks. Current Biology, 25(11):1426-1436, Jun 2015. ISSN 09609822. doi: 10.1016/j.cub.2015.04.005.

47. Kai Liu, Juhyun Kim, Dong Won Kim, Yi Stephanie Zhang, Hechen Bao, Myrto Denaxa, Szu-Aun Lim, Eileen Kim, Chang Liu, Ian R. Wickersham, and et al. Lhx6-positive gaba-releasing neurons of the zona incerta promote sleep. Nature, 548(7669):582-587, Aug 2017. ISSN 0028-0836. doi: 10.1038/nature23663.

48. N. R. Wall, I. R. Wickersham, A. Cetin, M. De La Parra, and E. M. Callaway. Monosynaptic circuit tracing in vivo through 
bioRxiv preprint doi: https://doi.org/10.1101/754812; this version posted September 3, 2019. The copyright holder for this preprint (which was not certified by peer review) is the author/funder, who has granted bioRxiv a license to display the preprint in perpetuity. It is made available under aCC-BY-ND 4.0 International license.

cre-dependent targeting and complementation of modified rabies virus. Proceedings of the National Academy of Sciences, 107(50): 21848-21853, Dec 2010. ISSN 0027-8424. doi: 10.1073/pnas. 1011756107.

49. Gidon Felsen and Zachary F. Mainen. Neural substrates of sensoryguided locomotor decisions in the rat superior colliculus. Neuron, 60(1):137-148, Oct 2008. ISSN 08966273. doi: 10.1016/j.neuron. 2008.09.019.

50. C. Shang, Z. Liu, Z. Chen, Y. Shi, Q. Wang, S. Liu, D. Li, and P. Cao. A parvalbumin-positive excitatory visual pathway to trigger fear responses in mice. Science, 348(6242):1472-1477, Jun 2015. ISSN 0036-8075. doi: 10.1126/science.aaa8694.

51. Brian Zingg, Xiao-lin Chou, Zheng-gang Zhang, Lukas Mesik, Feixue Liang, Huizhong Whit Tao, and Li I. Zhang. Aav-mediated anterograde transsynaptic tagging: Mapping corticocollicular inputdefined neural pathways for defense behaviors. Neuron, 93(1): 33-47, Jan 2017. ISSN 08966273. doi: 10.1016/j.neuron.2016.11. 045.

52. Ana F. Oliveira and Keisuke Yonehara. The mouse superior colliculus as a model system for investigating cell type-based mechanisms of visual motor transformation. Frontiers in Neural Circuits, 12:59, Jul 2018. ISSN 1662-5110. doi: 10.3389/fncir.2018.00059.

53. Lotta Borgius, C. Ernesto Restrepo, Richardson N. Leao, Noor Saleh, and Ole Kiehn. A transgenic mouse line for molecular genetic analysis of excitatory glutamatergic neurons. Molecular and Cellular Neuroscience, 45(3):245-257, Nov 2010. ISSN 10447431. doi: 10.1016/j.mcn.2010.06.016.

54. P Fagerstedt and F Ullén. Lateral turns in the lamprey. i. patterns of motoneuron activity. Journal of neurophysiology, 86(5):2246-56, Nov 2001. ISSN 0022-3077. doi: 10.1152/jn.2001.86.5.2246.

55. Patriq Fagerstedt, Grigori N. Orlovsky, Tatiana G. Deliagina, Sten Grillner, and Fredrik Ullén. Lateral turns in the lamprey. ii. activity of reticulospinal neurons during the generation of fictive turns. Journal of Neurophysiology, 86(5):2257-2265, Nov 2001. ISSN 0022-3077. doi: $10.1152 /$ jn.2001.86.5.2257.

56. Sten Grillner, Alexander Kozlov, Paolo Dario, Cesare Stefanini, Arianna Menciassi, Anders Lansner, and Jeanette Hellgren Kotaleski. Modeling a vertebrate motor system: pattern generation, steering and control of body orientation, volume 165, page 221-234. 2007. doi: 10.1016/S0079-6123(06)65014-0.

57. Kuo Hua Huang, Misha B Ahrens, Timothy W Dunn, and Florian Engert. Spinal projection neurons control turning behaviors in zebrafish. Current biology: $C B, 23(16): 1566-73$, Aug 2013. ISSN 1879-0445. doi: 10.1016/j.cub.2013.06.044.

58. Alexander K Kozlov, Andreas A Kardamakis, Jeanette Hellgren Kotaleski, and Sten Grillner. Gating of steering signals through phasic modulation of reticulospinal neurons during locomotion. Proceedings of the National Academy of Sciences of the United States of America, 111(9):3591-6, Mar 2014. ISSN 1091-6490. doi: 10.1073/pnas.1401459111.

59. Tod R Thiele, Joseph C Donovan, and Herwig Baier. Descending control of swim posture by a midbrain nucleus in zebrafish. Neuron, 83(3):679-91, Aug 2014. ISSN 1097-4199. doi: 10.1016/j.neuron. 2014.04.018.

60. Eyal Gruntman, Yoav Benjamini, and Ilan Golani. Coordination of steering in a free-trotting quadruped. Journal of comparative physiology. A, Neuroethology, sensory, neural, and behavioral physiology, 193(3):331-45, Mar 2007. ISSN 0340-7594. doi: 10.1007/ s00359-006-0187-5.

61. Martha W. Bagnall, Brian Zingg, Alexandra Sakatos, Setareh H. Moghadam, Hanns Ulrich Zeilhofer, and Sascha du Lac. Glycinergic projection neurons of the cerebellum. Journal of Neuroscience, 29(32):10104-10110, Aug 2009. ISSN 0270-6474. doi: 10.1523/JNEUROSCI.2087-09.2009.

62. Tomaso Muzzu, Susanna Mitolo, Giuseppe P. Gava, and Simon R. Schultz. Encoding of locomotion kinematics in the mouse cerebellum. PLOS ONE, 13(9):e0203900, Sep 2018. ISSN 1932-6203. doi: 10.1371/journal.pone.0203900.

63. Eiman Azim, Juan Jiang, Bror Alstermark, and Thomas M. Jessell.
Skilled reaching relies on a v2a propriospinal internal copy circuit. Nature, 508(7496):357-363, Feb 2014. ISSN 0028-0836. doi: 10. 1038/nature 13021

64. Carmelo Bellardita, Vittorio Caggiano, Roberto Leiras, Vanessa Caldeira, Andrea Fuchs, Julien Bouvier, Peter Löw, and Ole Kiehn. Spatiotemporal correlation of spinal network dynamics underlying spasms in chronic spinalized mice. eLife, 6:e23011, Feb 2017. ISSN 2050-084X. doi: 10.7554/eLife.23011.

65. Seung Wook Oh, Julie A. Harris, Lydia Ng, Brent Winslow, Nicholas Cain, Stefan Mihalas, Quanxin Wang, Chris Lau, Leonard Kuan, Alex M. Henry, and et al. A mesoscale connectome of the mouse brain. Nature, 508(7495):207-214, Apr 2014. ISSN 0028-0836. doi: 10.1038/nature13186.

66. Andrew J Murray, Jonas-Frederic Sauer, Gernot Riedel, Christina McClure, Laura Ansel, Lesley Cheyne, Marlene Bartos, William Wisden, and Peer Wulff. Parvalbumin-positive ca1 interneurons are required for spatial working but not for reference memory. Nature Neuroscience, 14(3):297-299, Mar 2011. ISSN 1097-6256. doi: 10.1038/nn.2751.

67. Ian R. Wickersham and Heather A. Sullivan. Rabies viral vectors for monosynaptic tracing and targeted transgene expression in neurons. Cold Spring Harbor Protocols, 2015. ISSN 15596095. doi: 10.1101/pdb.prot072389.

68. Keith B. J. Franklin and George Paxinos. Paxinos and Franklin's The mouse brain in stereotaxic coordinates. ISBN 9780123910578.

69. Tanmay Nath, Alexander Mathis, An Chi Chen, Amir Patel, Matthias Bethge, and Mackenzie Weygandt Mathis. Using deeplabcut for 3d markerless pose estimation across species and behaviors. Nature Protocols, 14(7):2152-2176, Jul 2019. ISSN 1754-2189. doi: 10. 1038/s41596-019-0176-0.

70. Ed S. Lein, Michael J. Hawrylycz, Nancy Ao, Mikael Ayres, Amy Bensinger, Amy Bernard, Andrew F. Boe, Mark S. Boguski, Kevin S. Brockway, Emi J. Byrnes, and et al. Genome-wide atlas of gene expression in the adult mouse brain. Nature, 445(7124):168-176, Jan 2007. ISSN 0028-0836. doi: 10.1038/nature05453. 


\section{MATERIALS AND METHODS}

Mice. All animal experiments and procedures were approved by Dyrefors $ø$ gstilsynet in Denmark and the local ethics committee at the University of Copenhagen. The Chxlo ${ }^{\text {Cre }}$ mouse is the same as that used previously (9, 63). The Vglut2 Cre mouse is described in (53). $R 26 R^{C h R 2-E Y F P}$ mice were obtained from Jackson Laboratories (Jackson Stock 012569). For in vitro experiments, we used newborn mice from $C h x 10^{C r e}$ and $R 26 R^{C h R 2-E Y F P}$ crosses. For in vivo experiments, we used hemizygous Chx $10^{\text {Cre }}$, Vglut $2^{\text {Cre }}$, or wild type mice (C57BL6/J (Jackson Stock 000664) greater than 8 weeks of age. Experiments were performed with similar numbers of male and female mice.

In Vitro Recording and Optogenetics. Locomotor-like activity was recorded from in vitro brainstem-spinal cord preparations isolated from P0-4 Chx $10^{C r e} ; R 26 R^{C h R 2-E Y F P}$ mice. Neonatal mice were anesthetized, and decapitated at the level of the midbrain. Mice were then eviscerated, and vertebral bodies were quickly removed ventrally from the level of the rostral pons caudally to the sacral spinal cord under ice-cold oxygenated dissection buffer $\left(95 \% \mathrm{O}_{2} / 5 \% \mathrm{CO}_{2}, 4{ }^{\circ} \mathrm{C}\right)$ composed of $111 \mathrm{mM}$ $\mathrm{NaCl}, 3 \mathrm{mM} \mathrm{KCl}, 26 \mathrm{mM} \mathrm{NaHCO}_{3}, 1.1 \mathrm{mM} \mathrm{KH}_{2} \mathrm{PO}_{4}, 0.25 \mathrm{mM} \mathrm{CaCl}_{2}, 3.7 \mathrm{mM} \mathrm{MgCl}_{2}$, and $11 \mathrm{mM}$ D-glucose. The ventral roots of the lumbar spinal cord were cut at their point of exit from the vertebral canal. The caudal neuroaxis was then isolated from the vertebral canal, and a coronal section was performed at the level of the facial motor nucleus (VII), rostral to the anterior inferior cerebellar artery. The rostral aspect of the preparation was maintained in an upward position to allow access for optical stimulation (9). Alternatively, the brainstem was split along the midline from the most rostral part to C1. The optical stimulation was then performed unilaterally from the cut surface.

A two-compartment system was used for pharmacological separation of the brainstem and lumbar spinal cord. The preparation was pinned to a Sylgard stage, and Vaseline was applied to the upper thoracic spinal cord at T8. A peristaltic pump was used to separately perfuse the rostral (brainstem) and caudal (lumbar spinal cord) compartments with oxygenated recording buffer $\left(95 \% \mathrm{O}_{2} / 5 \% \mathrm{CO}_{2}, 22-24{ }^{\circ} \mathrm{C}\right.$ ), composed of $111 \mathrm{mM} \mathrm{NaCl}, 3 \mathrm{mM} \mathrm{KCl}, 26 \mathrm{mM} \mathrm{NaHCO} 3,1.1 \mathrm{mM} \mathrm{KH}_{2} \mathrm{PO}_{4}, 2.5 \mathrm{mM} \mathrm{CaCl}_{2}$, $1.25 \mathrm{mM} \mathrm{MgCl}$, and $11 \mathrm{mM}$ D-glucose. Kynurenic acid (KA, $4 \mathrm{mM}$ ) was applied to the brainstem compartment to block all glutamatergic transmission in the brainstem thereby isolating the contribution of Chx10 reticulospinal neurons to spinal circuits (9). A cocktail of serotonin (5HT, $8 \mu \mathrm{M}$ ) and N-methyl-D-aspartic acid (NMDA, $8 \mu \mathrm{M}$ ) was applied to the lumbar spinal cord compartment to initiate locomotor-like activity. Fast green dye was maintained within the rostral compartment to verify an intact diffusion barrier, that is, drugs from the rostral compartment did not mix with the caudal compartment and vice versa.

Bipolar suction electrodes were attached to the right and left L2 ventral roots. The signal was amplified 5000-10000 times, band-pass filtered from $100 \mathrm{~Hz}$ to $1 \mathrm{kHz}$ and sampled at a frequency of $1 \mathrm{kHz}$.

Optogenetic stimulation of ChR2-expressing neurons was performed using a $473 \mathrm{~nm}$ laser system (UGA-40; Rapp Optoelectronic), which delivered blue light at an intensity of $30 \mathrm{~mW} \mathrm{~mm}^{-2}$ (64). Blue light was directed at the preparation using an optical fiber ( $200 \mu \mathrm{m}$ core, $0.22 \mathrm{NA}$, Thorlabs). Photo illumination was carried out continuously for 5-15 s. Burst amplitude (Figure 2D) was analyzed from rectified, integrated (bin $=0.25 \mathrm{~s}$ ) traces, where we quantified 5 bursts before, those bursts during, and 5 bursts after light stimulation. 1-6 trials were quantified for each animal.

Stereotaxic Injections. Viral injections in adult mice were performed using a stereotactic injection system (Neurostar, Tübingen, Germany). Mice were anesthetized with $4 \%$ isofluorane, and maintained under anesthesia with $2 \%$ isofluorane for the duration of the surgery. Anesthetic depth was verified using a toe-pinch test. Virus was mixed with fast green for visualization, and injected using a glass micropipette at a rate of $100 \mathrm{nl} \mathrm{min}^{-1}$. The glass micropipette was held in place for 5 min following injection to prevent backflow.

For unilateral anterograde tracing from Gi to the spinal cord, we injected $250 \mathrm{nl}$ AAV1-phSyn1(S)-FLEX-tdTomato-T2ASypEGFP-WPRE (5.56x10 $11 \mathrm{ml}^{-1}$, Viral Vector Core, Salk Institute for Biological Sciences; Addgene 51509) (65) in Chxl0 Cre animals at least 8 weeks of age. Mice were perfused 6 weeks after the injection. All injections in Gi were made at $6.0 \mathrm{~mm} \mathrm{AP,}$ $\pm 0.8 \mathrm{~mm} \mathrm{ML}$, and $5.5 \mathrm{~mm}$ DV relative to bregma.

For experiments using excitatory hM3Dq-DREADDs, Chx $10^{\text {Cre }}$ animals at least 8 weeks of age were injected in either the left or right Gi with $500 \mathrm{nl}$ of AAV5-hSyn1-DIO-hM3D(Gq)-mCherry-WPRE $\left(6 \times 10^{12} \mathrm{ml}^{-1}\right.$, Viral Vector Facility, University of Zurich, v89) or $500 \mathrm{nl}$ AAV5-hSyn1-DIO-mCherry control virus $\left(1.3 \times 10^{13} \mathrm{ml}^{-1}\right.$, Viral Vector Facility, University of Zurich, v84). Experiments were performed 3-6 weeks after injection. For in vivo optogenetics experiments, Chx $10^{C r e}$ animals were injected with $500 \mathrm{nl}$ of AAVdj-Ef1a-DIO-hChR2(E123T/T159C)-P2A-mCherry-WPRE in the left or right Gi. Optical fibers were implanted 3 weeks later at $-6 \mathrm{~mm}$ AP, $\pm 0.5 \mathrm{~mm} \mathrm{ML},-4.8 \mathrm{~mm}$ DV relative to bregma, and photostimulation experiments were performed the next day. For experiments using tetanus toxin virus, we injected either 300 or $500 \mathrm{nl}$ of AAV1-FLEX-TeLCEGFP to inhibit $C h \times 10$ Gi neurons $(9,66)$, or $500 \mathrm{nl}$ AAV5-FLEX-EGFP as a control. Tetanus toxin behavioral experiments were performed within 9 days of viral injection. For inhibitory DREADDs, Chx $10^{C r e}$ mice were injected with $350 \mathrm{nl}$ of AAVFLEX-hM4Di-mCherry in the left or right Gi $\left(7.4 \times 10^{12} \mathrm{ml}^{-1}\right.$, Viral Vector Facility, University of Zurich, v84). Experiments were performed 3-6 weeks after injection. 
For rabies transsynaptic labeling, the left Gi was injected with $200 \mathrm{nl}$ of a 1:1 mixture of AAV-syn-FLEX-splitTVA-EGFPtTA (Addgene 100798) and AAV-TREtight-mTagBFP2-B19G (Addgene 100799) (?). Seven days later, we injected $500 \mathrm{nl}$ of RV $\Delta \mathrm{G}-4 \mathrm{mCherry}(\mathrm{EnvA})$ in the same location. Mice were perfused 7 days after the second injection. The synthesis of pAAV-syn-FLEX-splitTVA-EGFP-tTA, pAAV-TREtight-mTagBFP2-B19G, and RV $\Delta \mathrm{G}-4 \mathrm{mCherry}(\mathrm{EnvA})$ has been described $(47,67)$.

For targeting the $\mathrm{SC}_{\text {contra- }}-\mathrm{Gi}_{\text {ipsi }}$ projection, injection of AAV-DIO-hM3Dq-mCherry or AAV5-dFRT-hM3Dq-mCherry $\left(3.5 \times 10^{12} \mathrm{ml}^{-1}\right.$, Viral Vector Facility, University of Zurich, v189-5) was followed 1 week by injection of $\mathrm{AAV}_{\text {retro-EGFP-Cre }}$ $\left(1.3 \times 10^{13} \mathrm{ml}^{-1}\right.$, Addgene, 105540-AAVrg), $\mathrm{AAV}_{\text {retro }}$-FLEX-EGFP-2A-FlpO $\left(6.8 \times 10^{12} \mathrm{ml}^{-1}\right.$, Viral Vector Facility, University

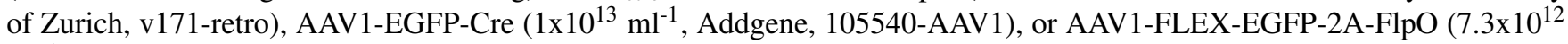
$\mathrm{ml}^{-1}$, Viral Vector Facility, University of Zurich, v171-1). Behavioral experiments were performed 1-2 weeks following second viral injection. For retrograde targeting of $\mathrm{SC}_{\text {contra }}-\mathrm{Gi}_{\text {ipsi }}$ projection neurons in wild-type mice, $80 \mathrm{nl}$ of AAV-DIO-hM3DqmCherry was injected in $\mathrm{SC}_{\text {contra }}$ and $150 \mathrm{nl}$ of $\mathrm{AAV}_{\text {retro }}$-EGFP-Cre in $\mathrm{Gi}_{\text {ipsi }}$. For anterograde targeting of $\mathrm{SC}_{\text {contra }}-\mathrm{Gi}_{\text {ipsi }}$ postsynaptic neurons in wild-type mice, $40 \mathrm{nl}$ of AAV1-EGFP-Cre was injected in $\mathrm{SC}_{\text {contra }}$ and $150 \mathrm{nl}$ of AAV-DIO-hM3Dq-mCherry in $\mathrm{Gi}_{i p s i}$. For retrograde targeting of $\mathrm{SC}_{\text {contra }}-\mathrm{Gi}_{\text {ipsi }}$ projection neurons in $V g l u t 2^{C r e}$ mice, $80 \mathrm{nl}$ of AAV-dFRT-hM3Dq-mCherry was injected in $\mathrm{SC}_{\text {contra }}$ and $150 \mathrm{nl}$ of $\mathrm{AAV}_{\text {retro }}$-FLEX-EGFP-2A-FlpO in $\mathrm{Gi}_{i p s i}$. For anterograde targeting of $\mathrm{SC}_{\text {contra }}-\mathrm{Gi}_{\text {ipsi }}$ Chx10 postsynaptic neurons in Chx10 Cre mice, $80 \mathrm{nl}$ of AAV1-FLEX-EGFP-2A-FlpO was injected in $\mathrm{SC}_{\text {contra }}$ and $500 \mathrm{nl}$ of AAV-dFRT-hM3Dq-mCherry in $\mathrm{Gi}_{\mathrm{ipsi}}$. SC coordinates used for these experiments were based on localization of RV $\Delta \mathrm{G}$ 4mCherry(EnvA) labeled cells (68): SC: $-3.5 \mathrm{~mm} \mathrm{AP,} \pm 1.12 \mathrm{~mm} \mathrm{ML}$, and $2.15 \mathrm{~mm}$ DV relative to bregma.

In Vivo Optogenetics. Optical fibers ( $200 \mu \mathrm{m}$ core, NA 0.22 , Thorlabs) were implanted 3 weeks after ChR2 viral injection (see information on injection/implantation above). Photostimulation experiments were carried out the day after implantation. Fibers were held in a $1.25 \mathrm{~mm}$ ferrule, and coupled to a $473 \mathrm{~nm}$ laser (Optoduet, Ikecool Corporation) via a ceramic mating sleeve. Photostimuli were manually triggered via a TTL-pulse given by Ethovision to a Master-8 pulse generator. Laser power was adjusted at $40 \mathrm{~Hz}(10 \mathrm{~ms}$ pulses for $1 \mathrm{~s}$ total duration) to initiate a strong turning response in each animal. We found that the laser power necessary to initiate a response was graded between $5-20 \mathrm{~mW}$.

Limb Dynamics. DeepLabCut $(45,69)$ was used for markerless extraction of paw position in an openfield. DeepLabCut 2.0 was installed on a PC equipped with a GEforce RTX 2080 Ti graphics card. We captured videos of mice moving from below (50 f.p.s.). Body center position was tracked using Ethovision XT, and video segments were extracted for locomotor bouts directed through the center of the arena that were $>5 \mathrm{~cm} \mathrm{~s}^{-1}$ and $>15 \mathrm{~cm}$ long. The four paws, the tip of the nose, and the base of the tail were tracked using DeepLabCut 2.0. 200 frames were randomly selected for labeling. The network was trained for 200,000 iterations until the loss reached a plateau. Segments of recording with likelihood $<0.6$ for all markers were excluded.

Subsequent analyses of limb kinematics were made using custom scripts in Python 3.7. The body angle was defined as tail base-nose angle relative to the $\mathrm{x}$ axis. The velocity of each paw was used to define steps. The beginning of the swing phase was defined as the point where paw velocity passed above $9 \mathrm{~cm} \mathrm{~s}^{-1}$, and the end of the swing phase (i.e., the beginning of the stance phase) was defined as the point where paw velocity passed back below $9 \mathrm{~cm} \mathrm{~s}^{-1}$. During alternating gates including fast walk and trot, animals move forward through the environment using diagonals-i.e. one forelimb and the contralateral hindlimb. Diagonal steps were defined as those occurring near simultaneously (within $0.1 \mathrm{~s}$ ) for diagonal limbs. For each paw, stride length was calculated as the length of the segment between the paw at the beginning and end of the diagonal step. We compared differences in stride length between the left and right sides with body angle at the beginning and end of a diagonal step. Pearson's correlation coefficient was used to estimate how stride length correlated with body angle.

Tissue Immunochemistry \& Imaging. Mice were euthanized by anesthetic overdose with pentobarbital $\left(250 \mathrm{mg} \mathrm{kg}^{-1}\right)$, and perfused transcardially with $4{ }^{\circ} \mathrm{C}$ saline followed by $4 \%$ paraformaldehyde. Brain and spinal cord tissue was dissected free, and then post-fixed in $4 \%$ paraformaldehyde for $3 \mathrm{~h}$ at $4{ }^{\circ} \mathrm{C}$. Tissue was cryoprotected by incubation in $30 \%$ sucrose in phosphate buffered saline (PBS) overnight. Tissue was then embedded in Neg-50 medium (ThermoFisher Scientific) for cryostat sectioning. Coronal or sagittal sections were obtained on a Leica cryostat and mounted on Superfrost Plus slides (ThermoFisher Scientific). Brainstem coronal sections were cut at $30 \mu \mathrm{m}$ thickness, whereas spinal cord coronal sections were cut at $20 \mu \mathrm{m}$. Sagittal sections were cut at $30 \mu \mathrm{m}$.

Sections were rehydrated for 5 minutes in PBS $+0.5 \%$ Triton-X100 (PBS-T; Sigma-Aldrich), and then blocked for $2 \mathrm{~h}$ in $10 \%$ normal donkey serum in PBS-T (Jackson ImmunoResearch). Sections were incubated overnight with primary antibodies diluted in blocking solution. We used the following primary antibodies: chicken anti-GFP (1:1000, Abcam, ab13970) and rabbit anti-DsRed/tdTomato/mCherry (1:1000, Clontech, 632496). Slides were washed 4 times in PBS-T, and then incubated with appropriate donkey secondary antibodies diluted in blocking solution (1:500, ThermoFisher Scientific). Slides were washed 4 times in PBS-T, counterstained with Hoechst 33342 (1:2000) or NeuroTrace 435 (1:400, ThermoFisher Scientific), and were mounted with coverslips using mowiol 4-88 medium. Sections were imaged using either a Zeiss widefield epifluorescence microscope or a Zeiss LSM 780 confocal microscope. 
Drugs. Clozapine-N-oxide (CNO, Tocris, 4936) was dissolved in saline immediately prior to behavioral experiments. CNO was administered intraperitoneally at a dose of $0.5 \mathrm{mg} \mathrm{kg}^{-1}$ (Chx10 Cre $>$ FLEX-hM3Dq; Figure 3, Figure S2) or $1 \mathrm{mg} \mathrm{kg}^{-1}$ (Chx10 ${ }^{\mathrm{Cre}}>$ FLEX-hM4Di, Figure 4; SC or Gi > FLEX-hM3Dq or dFRT-hM3Dq, Figure 7).

Cylinder Test. Mice were placed in a $15 \mathrm{~cm}$ diameter cylinder for 10 minutes. Ethovision XT (Noldus) software was used to capture video (15 f.p.s.) and carry out tracking of head, center point, and tail. $360^{\circ}$ clockwise and counterclockwise revolutions were quantified (tail point to center point, $50^{\circ}$ threshold).

Open Field. Mice were acclimated to the behavioral suite prior to testing in an open field arena. Open field analysis was carried out in a 50 x $50 \mathrm{~cm}$ square arena illuminated with an infrared lamp. Ethovision XT (Noldus) software was used to capture video (15-25 f.p.s.) and carry out tracking analysis. Mice were tracked using head, center, and tail points, enabling quantification of rotations as a function of time. Movement parameters taken in the open field included total movement (m), ambulation (time moving at a velocity greater than $2 \mathrm{~cm} \mathrm{~s}^{-1}$ ), and $360^{\circ}$ revolutions (tail point to center point, $50^{\circ}$ threshold). Tracking was carried out for 60 minutes. Mice were allowed to acclimate to the arena for $30 \mathrm{~min}$, followed by a $30 \mathrm{~min}$ probe. Movement parameters are reported for minutes $30-60$.

Ipsilateral/Contralateral Spiral Maze. Spiral-shaped mazes were used to test clockwise/counterclockwise movement preference. For this purpose, mazes were fabricated which could be explored from the center-point to the periphery only by moving in a clockwise or counterclockwise direction (see Figures $6 \mathrm{~A}$ and $6 \mathrm{C}$ for illustration). These mazes exhibit an increasing radius as mice move from the center-point to the periphery. For each mouse, a 10 minute trial was conducted in either the left- or right-turned maze. One affected mouse exhibited a turning radius that was smaller than the center of the maze (see Figures 6A and 6C). This affected mouse was excluded from analysis because it could not complete either the ipsilateral or contralateral maze. Body tracking was carried out with Ethovision XT (Noldus) using center-point detection.

Analysis. For quantification of viral transduction in $\mathrm{Gi}$, the number of neurons ipsilateral and contralateral to the injection site was counted in every 10th section. These counts were used to estimate the percentage of viral transduction contained to the ipsilateral side (Figure 1). Similar analysis was performed to estimate the percentage of labeled neurons in the ipsilateral versus contralateral superior colliculus (Figure 7).

For analysis of synGFP ${ }^{+}$punctae in tdTom-2A-synGFP traced projections, high resolution images of GFP-stained coronal spinal cord sections were obtained with a confocal microscope. Three images from each spinal level (i.e. cervical, thoracic, lumbar) were quantified from three mice. Analysis of synGFP punctae was restricted to the gray matter, with the assumption that synGFP ${ }^{+}$punctae in white matter reflected transport of synGFP protein rather than bona fide synapses. SynGFP punctae were isolated from the images using thresholding, and the position of each punctum was extracted using the particle analysis feature in ImageJ (https://imagej.nih.gov/ij/). The position of the central canal was defined using the Nissl counterstain, and synGFP ${ }^{+}$punctum position was normalized between sections and animals using this point as a reference. From this data set, the percentage of synapses ipsilateral and contralateral to the injection site was estimated (Figure 1). Synaptic density plots were constructed in R, where gradation represents the range from zero to maximum density at each spinal level (Figure 1G). Synaptic density plots represent the average of 3 animals. Coordinates and abbreviations are based on Paxinos and Franklin's reference atlas (68), and corresponding plates are from the Allen Mouse Brain Atlas (70).

Statistics. A two-tailed t-test was performed for pairwise comparisons. For multiple comparisons, a one-way ANOVA was performed to determine whether significant differences existed between conditions, followed by Tukey-Kramer HSD for assigning levels of significance. All data are given as mean \pm standard error mean. Individual data points are plotted for every analysis. For each comparison, information on statistical analyses, $P$ value statistics, and $n$ values are listed in the figure legend. $n$ values represent distinct biological replicates. $P<0.05$ was considered statistically significant, where $* P<0.05, * * P<0.01$, and $* * * P<0.001$. 

under aCC-BY-ND 4.0 International license.

\section{Supplementary Material}

A

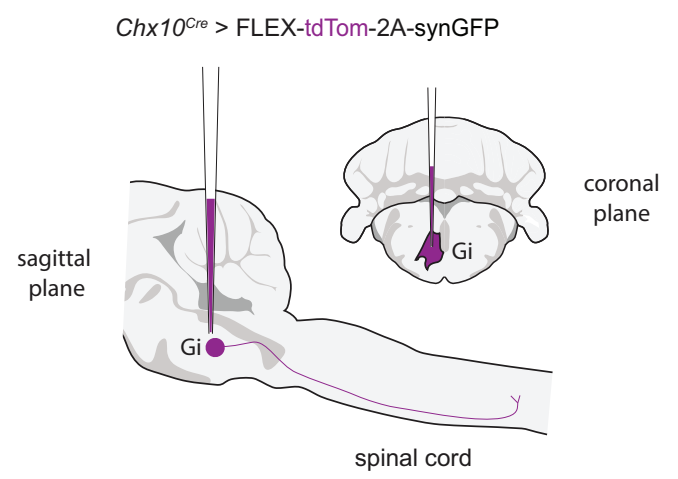

B
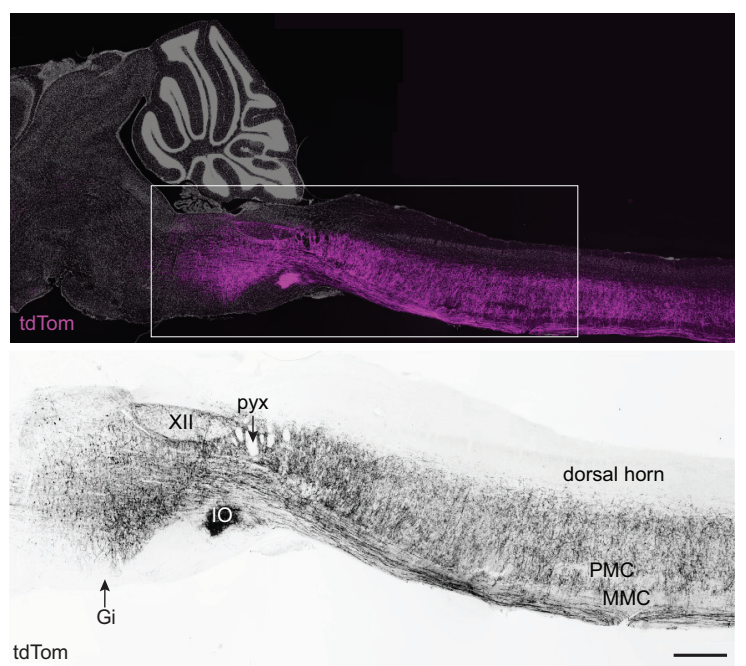

C
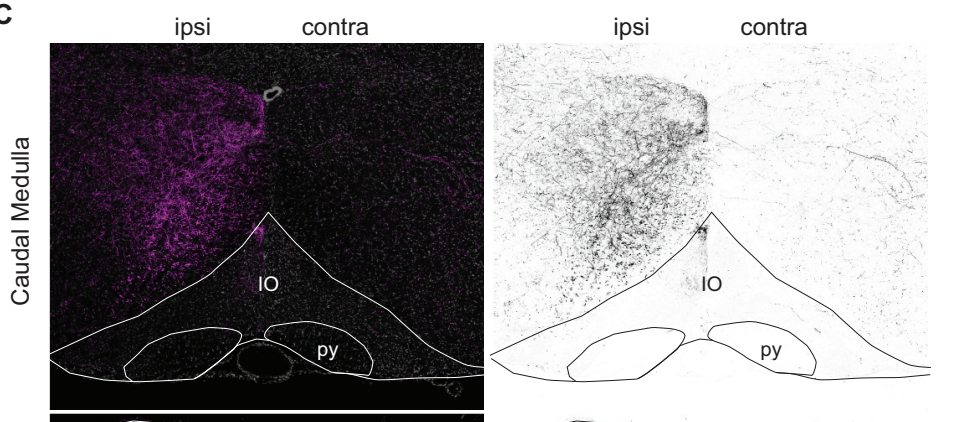

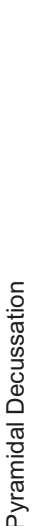

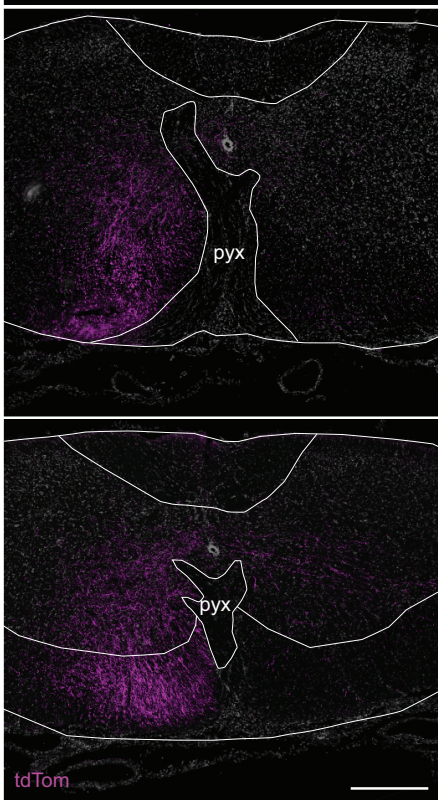

Figure S1. Chx10 Gi neurons form a prominent tract of ipsilaterally projecting axons. A, Unilateral labeling of Chx10 neurons of the rostral gigantocellularis (Gi) using the Cre-dependent anterograde tracer AAV-FLEX-tdTom-2A-synGFP. B, Top, Sagittal section of tdTom $^{+}$projections ipsilateral to the injection site. tdTom ${ }^{+}$axons formed a prominent tract that projected caudally to the spinal cord. Bottom, inset from Top. Scale bar $=500 \mu \mathrm{m}$. XII, hypoglossal motor nucleus; pyx, pyramidal decussation; IO, inferior olive; PMC, phrenic motor column; MMC, medial motor column. C, Coalescence of Chx10 reticulospinal axons dorsal to the inferior olive (top), and subsequent positioning in the ventrolateral funiculus at the level of the pyramidal decussation (bottom). 
A Chx10 cre $>$ FLEX-mCherry

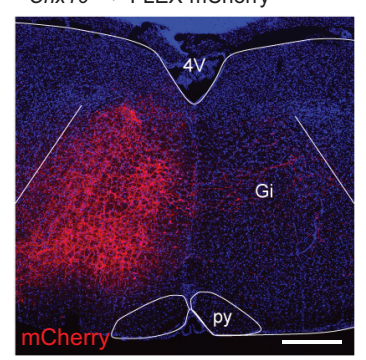

B

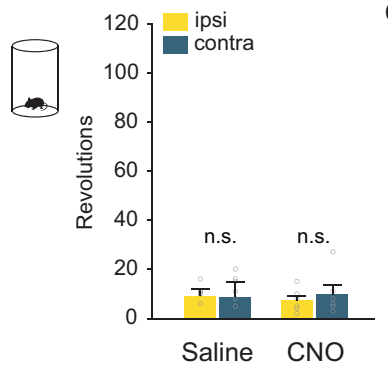

C

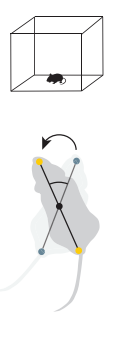

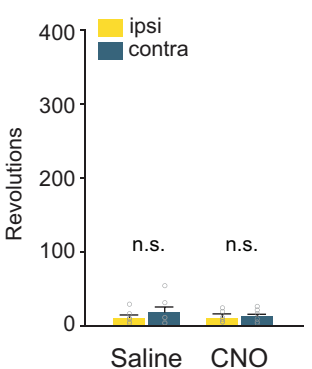

Figure S2. CNO administration does not affect turning preference in control AAV-mCherry injected mice. A, Example of mCherry expression 3 weeks after a $500 \mathrm{nl}$ unilateral injection of AAV-FLEX-mCherry in a Chx $10^{\text {Cre }}$ mouse. mCherry expression is confined to the side ipsilateral to injection. B, Turning preference in a 10 minute cylinder assay is unaffected by administration of CNO. $P>0.45$, one-way ANOVA with Tukey HSD, $n=6$. C, Turning preference in an open field arena is unaffected by administration of CNO. $P>0.77$, one-way ANOVA with Tukey HSD, $n=6$. D, Instantaneous quantification of ipsilateral and contralateral revolutions after injection of either saline or CNO in AAV-FLEX-mCherry injected mice. 
A

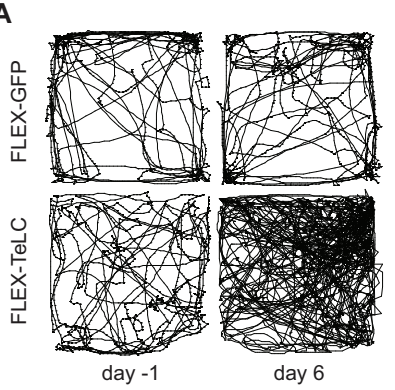

B

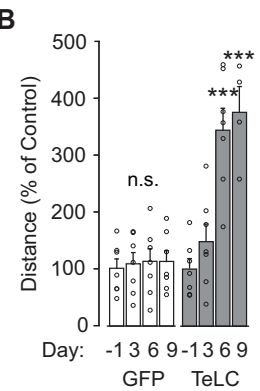

C

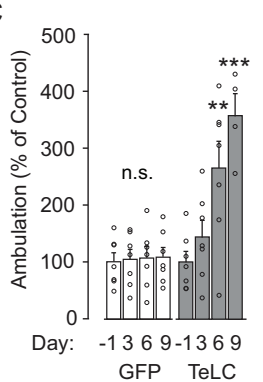

Figure S3. Unilateral silencing of Chx10 Gi neurons increases locomotion in an open field assay. A, Open field analysis of a Chx10 Cre $>$ FLEX-GFP or FLEX-TeLC mouse one day before AAV injection (day -1) and 6 days after AAV injection. Traces represent 15 minutes of open field activity. B, Total distance traveled during a 30 minute open field trial -1 , 3,6 , or 9 days after AAV injection as a percentage of day -1. GFP: $P>0.97$ for each comparison, $n=7$. TeLC: $* * P<1.2 \times 10^{-4}$ (day 6 and 9 vs. -1 ), $n=7$ for days $-1,3$, and 6, $n=4$ for day 9. One-way ANOVA with Tukey HSD. C, Open field ambulation as a percentage of day -1 . GFP: $P>0.98$ for each comparison. TeLC: $* * P=0.009$, day 6 vs. $-1 ; * * * P=6.6 \times 10^{-4}$, day 9 vs. -1 . One-way ANOVA with Tukey HSD. 


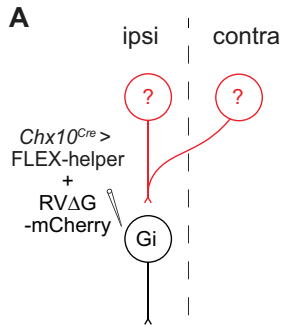

B
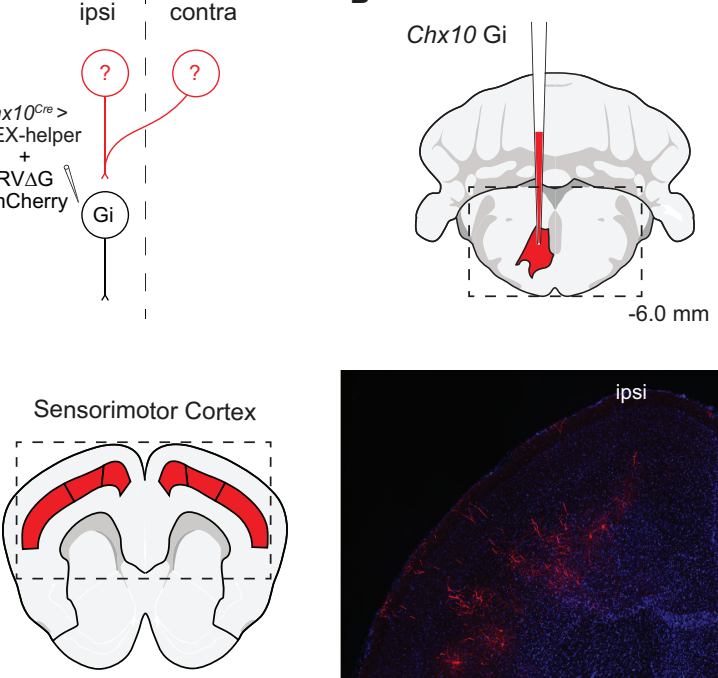

$+1.5 \mathrm{~mm}$

$\mathbf{F}$

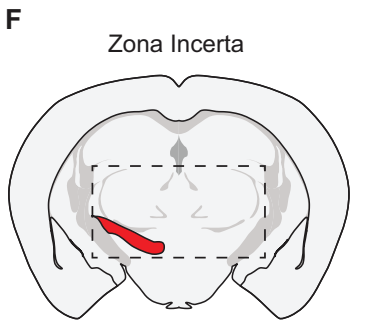

$-2.8 \mathrm{~mm}$

Hesencephalic Reticular

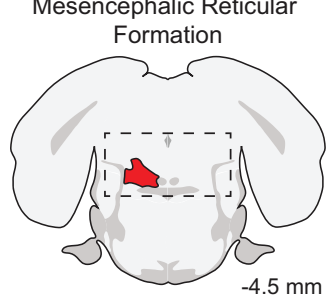

$J$

Deep Cerebellar Nuclei

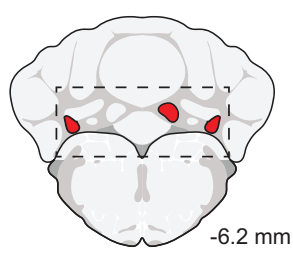

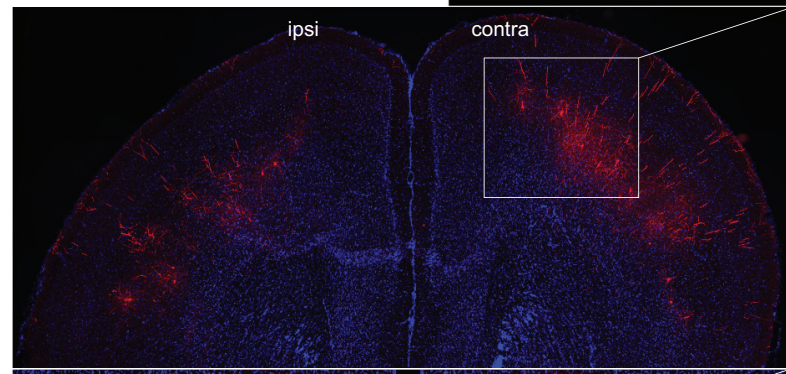
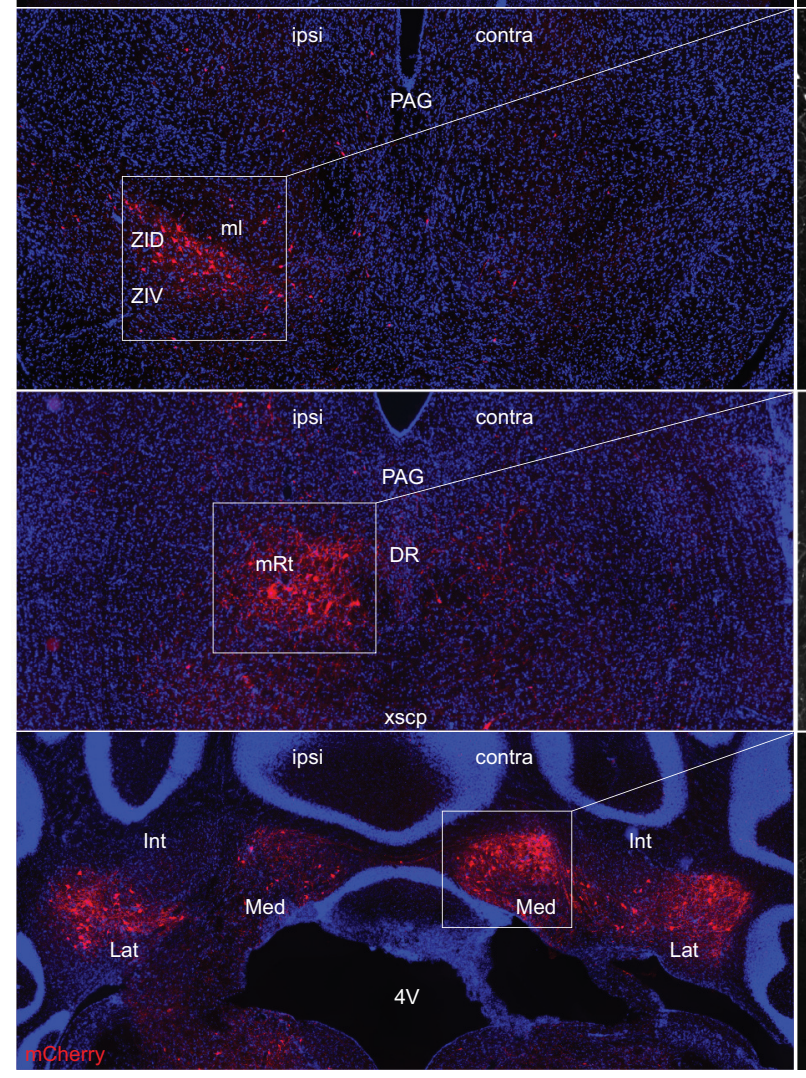

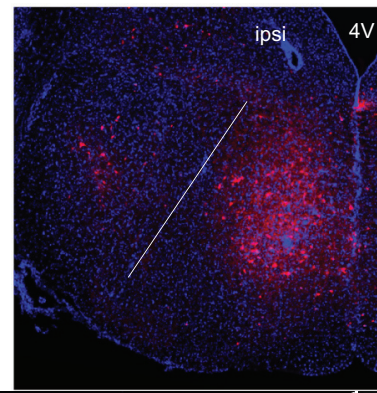

$4 \mathrm{~V}$ contra
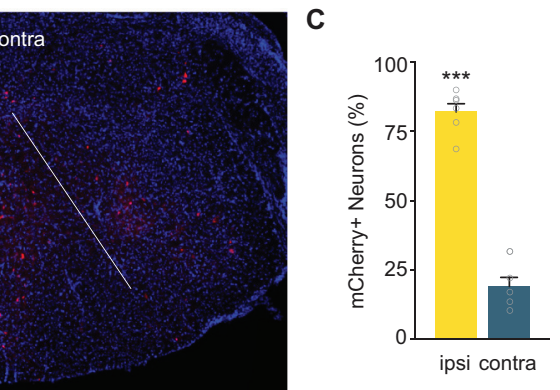

E

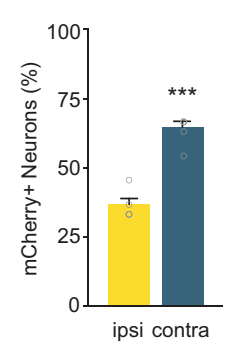

G
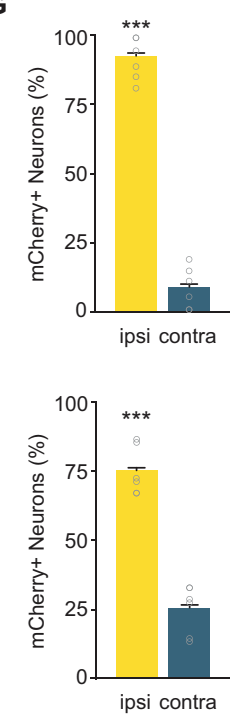

K

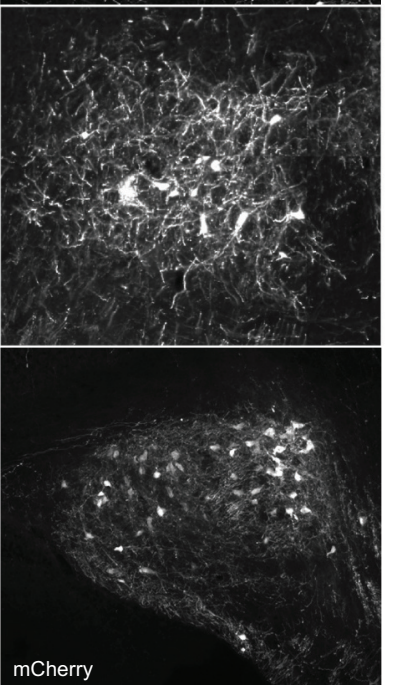

Figure S4. Monosynaptic rabies tracing identifies Chx10 Gi presynaptic inputs. A, A rabies transsynaptic tracing approach was used to identify presynaptic inputs to ChxlO Gi neurons (see also, Figure 7 and Table S1). B, Injection site in rostral Gi. Initial site of infection is visualized as a large population of mCherry ${ }^{+}$neurons, accompanied by dense mCherry ${ }^{+}$ processes. Starter neurons of the ipsilateral Gi do not exhibit a strong input from neurons of the contralateral Gi. C, Quantification of mCherry-labeled neurons in the ipsilateral and contralateral Gi. $* * * P=2.5 \times 10^{-7}$, two-tailed t-test, $n=6$. D, Bilateral input to Chx10 Gi neurons from neurons of primary motor and somatosensory cortex. Open yellow triangles point to soma from pyramidal neurons. Yellow asterisk indicates an apical dendrite. E, Quantification of mCherry-labeled neurons in the ipsilateral and contralateral cortex. $* * * P=1.9 \times 10^{-6}$, two-tailed t-test, $n=6$. F, Input to Chx 10 Gi neurons from neurons of the ipsilateral zona incerta. Presynaptic neurons were observed primarily in the dorsal (ZID) and caudal aspects of the zona incerta. G, Quantification of mCherry-labeled neurons in the ipsilateral and contralateral zona incerta. $* * * P=3.7 \times 10^{-9}$, two-tailed t-test, $n=6$. H, Unilateral input to Chx 10 Gi neurons from the ipsilateral mesencephalic reticular formation. I, Quantification of mCherry-labeled neurons in the ipsilateral and contralateral mesencephalic reticular formation. ${ }^{* * *} P=1.8 \times 10^{-6}$, two-tailed 
t-test, $n=6$. J, Medial (Med) and lateral (Lat) deep cerebellar nuclei exhibited unilateral or bilateral, respectively, input to Chx10 Gi neurons. K, Quantification of mCherry-labeled neurons in the deep cerebellar nuclei. Med, $* * * P=1.1 \times 10^{-5}$, two-tailed t-test, $n=6$; Lat, $P=0.56$, two-tailed t-test, $n=6$. 
Table S1. Summary of monosynaptic rabies tracing data.

\begin{tabular}{|c|c|c|c|c|c|c|c|}
\hline \multicolumn{4}{|c|}{ Cortex $($ Bregma $+2.2 \rightarrow-0.3 \mathrm{~mm})$} & \multicolumn{4}{|c|}{ Pons $(-4.7 \rightarrow-5.7 \mathrm{~mm})$} \\
\hline \multicolumn{2}{|c|}{ Ipsilateral } & \multicolumn{2}{|c|}{ Contralateral } & \multicolumn{2}{|c|}{ Ipsilateral } & \multicolumn{2}{|c|}{ Contralateral } \\
\hline M1 & +++ & M1 & +++ & $\mathrm{PnC}$ & ++ & $\mathrm{PnC}$ & + \\
\hline S1HL/FL & +++ & S1HL/FL & +++ & Irt & ++ & Irt & ++ \\
\hline S1DZ-J & ++ & S1DZ-J & ++ & PCRtA & + & PCRtA & + \\
\hline M2 & + & M2 & + & MveMC & + & MveMC & + \\
\hline Fr3 & + & Fr3 & + & SubCD & + & SubCD & + \\
\hline & & & & $\mathrm{PnO}$ & + & $\mathrm{PnO}$ & ++ \\
\hline \multicolumn{4}{|c|}{ Amygdala $(-0.2 \rightarrow-1.8 \mathrm{~mm})$} & $\mathrm{mRt}$ & +++ & $\mathrm{mRt}$ & + \\
\hline \multicolumn{2}{|c|}{ Ipsilateral } & \multicolumn{2}{|c|}{ Contralateral } & $\mathrm{PrCnF}$ & ++ & $\mathrm{PrCnF}$ & + \\
\hline $\mathrm{CeL} / \mathrm{C} / \mathrm{M}$ & ++ & & & & & $5 \mathrm{~N}$ & + \\
\hline BLA & + & & & & & & \\
\hline EAC & + & EAC & + & \multicolumn{4}{|c|}{ Cerebellum $(-5.7 \rightarrow-6.8 \mathrm{~mm})$} \\
\hline AA & + & & & \multicolumn{2}{|c|}{ Ipsilateral } & \multicolumn{2}{|c|}{ Contralateral } \\
\hline & & & & Med & + & Med & ++++ \\
\hline \multicolumn{4}{|c|}{ Zona Incerta $(-1.8 \rightarrow-2.8 \mathrm{~mm})$} & Lat & ++++ & Lat & +++ \\
\hline \multicolumn{2}{|c|}{ Ipsilateral } & \multicolumn{2}{|c|}{ Contralateral } & & & & \\
\hline ZID & +++ & ZID & + & \multicolumn{4}{|c|}{ Medulla $(-5.7 \rightarrow-7.8 \mathrm{~mm})$} \\
\hline ZIV & + & & & \multicolumn{2}{|c|}{ Ipsilateral } & \multicolumn{2}{|c|}{ Contralateral } \\
\hline & & & & MdV & + & $\mathrm{MdV}$ & ++ \\
\hline \multicolumn{4}{|c|}{ Midbrain/Superior Colliculus $(-3.1 \rightarrow-4.2 \mathrm{~mm})$} & Irt & ++ & Irt & +++ \\
\hline \multicolumn{2}{|c|}{ Ipsilateral } & \multicolumn{2}{|c|}{ Contralateral } & $\mathrm{MdD}$ & + & MdD & + \\
\hline InG & + & InG & +++ & LRt & + & LRt & ++ \\
\hline InW & ++ & InW & +++ & $* \mathrm{Gi}$ & +++ & $\mathrm{Gi}$ & + \\
\hline & & DpW & ++ & PCRt/A & ++ & PCRt/A & + \\
\hline $\mathrm{mRt}$ & ++ & $\mathrm{mRt}$ & + & LPGi & + & LPGi & + \\
\hline $\operatorname{PrCnF}$ & + & & & & & DPGi & ++ \\
\hline & & RMC & + & & & GiA & + \\
\hline
\end{tabular}

*site of primary infection

+ denotes presence of rabies mCherry ${ }^{+}$neurons

++ small/intermediate number of mCherry $^{+}$neurons

+++ significant population of mCherry ${ }^{+}$neurons

See also, Figure 7 and Figure S4. Qualitative analysis is based on curation of rabies retrograde tracing data from $n=$ 6 animals. Coordinates represent the rostrocaudal extent to which mCherry ${ }^{+}$neurons were observed in each corresponding structure (e.g. Cortex, Amygdala, etc.). Coordinates and abbreviations are based on Paxinos and Franklin's reference atlas (68).

Abbreviations: 5N, motor trigeminal nucleus; AA, anterior amygdaloid area; BLA, basolateral amygdaloid nucleus, anterior part; $\mathrm{CeC} / \mathrm{L} / \mathrm{M}$, central amygdaloid nucleus, capsular/lateral//medial division; DPGi, dorsal paragigantocellular nucleus; DpW, deep white layer of the superior colliculus; EAC, extended amygdala, central part; Fr3, frontal cortex, area 3; Gi/A, gigantocellular reticular nucleus, alpha part; InG/W, intermediate gray/white layer of the superior colliculus; IRt, intermediate reticular nucleus; Lat, lateral cerebellar nucleus; LPGi, lateral paragigantocellular nucleus; LRt, lateral reticular nucleus; M1, primary motor cortex; M2, secondary motor cortex; MdD/V, medullary reticular nucleus, dorsal/ventral part; Med, medial cerebellar nucleus; mRt, mesencephalic reticular formation; MVeMC, medial vestibular nucleus, magnocellular part; PCRt/A, parvicellular reticular nucleus, alpha part; $\mathrm{PnC} / \mathrm{O}$, pontine reticular nucleus, caudal/oral part; $\mathrm{PrCnF}$, precuneform area; $\mathrm{RMC}$, red nucleus, magnocellular part; S1 DZ/J/HL/FL, primary somatosensory cortex, dysgranular zone/jaw region/hindlimb region/forelimb region, SubCD, subcoeruleus nucleus, dorsal part; ZID/V, zona incerta, dorsal/ventral part. 
Video S1. Open field analysis of a left Gi Chx10 ${ }^{\text {Cre }}>$ FLEX-hM3Dq targeted mouse 0, 15, and 25 min after administration of CNO. Immediately following CNO administration $(0 \mathrm{~min})$, the mouse exhibits no preference in locomotor direction. After $15 \mathrm{~min}$, the mouse exhibits a clear preference toward the ipsilateral side, making large left-turn circles in the arena. After 25 min, the mouse exhibits sharp rotations toward the ipsilateral side. Playback is three times real speed.

Video S2. Photostimulation of left Gi Chx10 ${ }^{\text {Cre }}>$ FLEX-ChR2 targeted mice during spontaneous locomotion and at rest.

Video S3. Open field analysis of a left Gi Chx10 ${ }^{\text {Cre }}>$ FLEX-TeLC targeted mouse. Before viral injection (day -1), the mouse exhibited no left or right preference in locomotor direction. Six days following viral injection, the mouse exhibits a clear locomotor preference toward the contralateral (right) side. Playback is three times real speed.

Video S4. DeepLabCut tracking of footfalls and movement trajectories in wild-type, Chx10 ${ }^{\text {Cre }}>$ FLEX-hM3Dq, and $\mathrm{Ch} \times 10^{\mathrm{Cre}}>$ FLEX-TeLC targeted mice. Footfalls are plotted when the limb velocity reaches $0 \mathrm{~cm} \mathrm{~s}^{-1}$. 